\title{
Perturbation methods in evolutionary spectral analysis for linear dynamics and equivalent statistical linearization
}

\author{
Thomas Canor ${ }^{\mathrm{a}, \mathrm{b}}$, Luca Caracoglia ${ }^{\mathrm{c}}$, Vincent Denoël ${ }^{\mathrm{a}, *}$ \\ ${ }^{a}$ Structural Engineering Division, University of Liège, Allée de la Découverte, 9, B52/3, 4000 Liège, Belgium \\ b National Fund for Scientific Research of Belgium, Belgium \\ c Department of Civil and Environmental Engineering, Northeastern University, 400 Snell Engineering Center, 360 Huntington Avenue, Boston, MA 02115, \\ United States
}

\section{A R T I C L E I N F O}

\section{Article history:}

Received 28 September 2015

Received in revised form

31 May 2016

Accepted 25 July 2016

Available online 28 July 2016

Keywords:

Evolutionary spectral analysis

Asymptotic expansion

Multiple scales

Nonlinear dynamics

Gaussian equivalent linearization

Wind engineering

Earthquake engineering

\begin{abstract}
A B S T R A C T
The analysis of large-scale structures subject to transient random loads, coherent in space and time, is a classic problem encountered in earthquake and wind engineering. The simulation-based framework is usually seen as the most convenient approach for both linear and nonlinear dynamics. However, the generation of statistically consistent samples of an excitation field remains a heavy computational task. In light of this, perturbation techniques are applied to develop and improve evolutionary spectral analysis.

Advantageously performed in a standard modal basis, this evolutionary spectral analysis for linear structures requires the computation of the modal impulse response matrix. However, this matrix has no general closed-form expression in the presence of modal coupling. We propose therefore to model it by an asymptotic approximation, obtained by the inverse Fourier transform of an asymptotic expansion of the modal transfer matrix of the structure. This latter expansion considers the modal coupling as a perturbation of a main decoupled system. This strategy leads to an expansion known in a closed-form. Finally, the semi-group property allows the use of an efficient recurrence relation to approximate the modal evolutionary transfer matrix, i.e. the evolutionary extension of the transfer matrix.

The asymptotic expansion-based method and the recurrence relation are then applied to nonlinear transient dynamics by using Gaussian equivalent linearization. This extension is formalized by a multiple timescales approach, allowing to consider a linearized structure, namely a time variant system, as piecewise linear time invariant depending on a statistical timescale. The proposed developments are finally illustrated on realistic civil engineering applications.
\end{abstract}

(c) 2016 Elsevier Ltd. All rights reserved.

\section{Introduction}

\subsection{Context and methods}

In civil engineering, structures are designed to resist random loadings, such as wind forces or ground acceleration during earthquakes. In some cases, these loadings are said to be nonstationary or transient, i.e. their statistical characteristics are timedependent. This property is well-known for earthquakes, as the phenomenon is usually modeled by three phases: build-up, plateau and decay. Moreover, it would be fallacious to limit wind analysis to stationary processes. For example, in the case of downbursts or thunderstorms, we indubitably need to take into account the time evolution of the mean wind velocity and the

\footnotetext{
* Corresponding author.

E-mail addresses: thomas.canor@gmail.com (T. Canor), lucac@coe.neu.edu (L. Caracoglia),v.denoel@ulg.ac.be (V. Denoël).
}

intensity of turbulence. In the context of risk analysis in civil engineering, an adequate design requires to compute the time evolution of the statistics of the structural response.

The development of suitable methods to perform transient analysis has attracted the attention of the research community in the last 30 years. For linear structures, an analytical approach based on Duhamel's convolution [12], also called pseudo-excitation approach [38], may be used, even though this method proves to be exclusively efficient for systems in which the modal expansion is capable of decoupling the dynamic equations. Actually, practitioners and engineers consider Monte Carlo simulations as the most convenient method to perform linear nonstationary analysis. As to nonlinear structures, Proppe et al. [50] show that Monte Carlo simulations [19,59] and the equivalent linearization [52] are the only two feasible methods to perform stochastic analysis of large-scale structures, especially in a nonstationary setting.

Other time domain methods have been developed for both linear and nonlinear analyses. For instance, the concept of stochastic integration scheme proposed by To $[64,65]$ has been 
recently improved by Tootkaboni [66] and applied to linear systems subject to non-white excitations or to nonlinear systems by considering them as piecewise linear. The question of nonstationary Gaussian equivalent linearization (GEL) has been also addressed interestingly by the team of Schuëller in [46,54,55], who proposed a method based on the Karhunen-Loéve (K-L) expansion of the excitation. Accordingly, the $\mathrm{K}-\mathrm{L}$ expansion of the modal state space vector of the structure is substituted into the linearized equation of motion. The $\mathrm{K}-\mathrm{L}$ decomposition can also be used when measured data are available [60], even if they are often difficult to obtain in wind and earthquake engineering. Following similar ideas, stochastic averaging techniques have been used to determine approximate closed-form expressions for some specific problem, e.g. concerning the use of nonlinear viscous dampers [67].

A main drawback of the simulation-based framework in risk quantification is the difficulty to deal with large dimensional coherent excitation fields. In wind engineering, the forces due to wind blowing on large structures are usually modeled as a spatially coherent excitation field [61], i.e. a set of random processes simultaneously depending on both time and space [25]. On the other hand, for long structures subject to earthquake loads, the ground accelerations measured at different supports are different, but not statistically independent [35,72]. Actually, neglecting the coherence within the field may lead to underestimation of the structural response, while assuming fully correlated processes may result in possible overestimation. In fact, the computational burden inherent to these approaches is mainly associated with the generation of consistent and accurate samples of the excitation fields.

In earthquake or wind engineering, the coherence within the loading can be adequately modeled by a full power spectral density (PSD) matrix. The coherence is a way to express the time correlation in the frequency domain, a reason for trying to keep on working in the frequency domain. In wind engineering, a spectral approach is usually chosen to perform buffeting analysis, as explained for instance in [20,39]. For linear stationary problems, the spectral approach is by far the most efficient one, since it just consists in matrix multiplications performed for selected frequencies and in an integration of these matrices over the frequency domain. These operations are naturally preceded by a modal projection. In this context, the spectral approach clearly outscores the simulation-based framework in terms of accuracy and efficiency. In many cases, consideration of the different timescales of the excitation and of the response make this approach yet more efficient, even in case of slight structural nonlinearities [15].

This work aims at applying, in a nonstationary setting, the spectral analysis to both linear and nonlinear structures in civil engineering. Within this context, a specific family of transient processes is considered: the evolutionary processes. Such an unsteady process may be described by the PSD of an embedded stationary process and a modulation time window. Actually, the evolutionary spectral (EvSp) analysis may be understood as a natural extension of the spectral analysis, since the former approach should necessarily tend to the latter one over large timescales, provided the intensity envelope remains constant in time. The evolutionary approach remains an elegant formulation of transient phenomena, for which evolutionary models are available, as for earthquakes or downbursts $[68,33,10,11,56,73]$. However, it requires to work in both time and frequency domains, contrary to the classical spectral analysis.

As source and ground, the stricto sensu EvSp analysis has been formalized by Priestley $[48,49]$. The first pertinent applications are due to Hammond for SDOF and MDOF systems [28,29]. Those contributions are only focused on linear time-invariant (LTI) systems. Although the spectral approach is recognized as the most suitable method to analyze large-dimensional linear structures subject to stationary loadings, the evolutionary spectral analysis has not encountered a real enthusiasm in the fields of engineering for some reasons explained hereinafter. Before, some mathematical statements about evolutionary processes are presented.

\subsection{Mathematical statements}

On a probability space $(\Theta, \mathfrak{F}, \mathbb{P})$, the equation of motion of a $n$ DOF nonlinear system is

$\mathbf{M y}+\mathbf{C} \dot{\mathbf{y}}+\mathbf{K y}+\mathbf{g}(\mathbf{y}, \dot{\mathbf{y}})=\mathbf{f}$,

where $\mathbf{M}, \mathbf{C}$ and $\mathbf{K}$ are the $n$-dimensional mass, damping and stiffness matrices of the system, respectively, $\mathbf{f}(t, \theta): \mathbb{R}^{+} \times \Theta \mapsto \mathbb{R}^{n}$ is the vector of random exogenous Gaussian forces and the dot symbol denotes the time derivative. The hypothesis of Gaussianity is used throughout this work, since random loading processes can often be described in this way in wind or earthquake engineering. The vector $\mathbf{y}(t, \theta): \mathbb{R}^{+} \times \Theta \mapsto \mathbb{R}^{n}$ gathers the nodal displacements expected to be non-Gaussian processes due to the nonlinear forces in the vector function $\mathbf{g}(\mathbf{y}, \dot{\mathbf{y}}): \mathbb{R}^{n} \times \mathbb{R}^{n} \mapsto \mathbb{R}^{n}$. With this formalism, the equation of motion is split into four contributions: inertial forces, internal linear forces, internal nonlinear forces and exogenous random forces. Discarding the nonlinear forces $\mathbf{g}(\mathbf{y}, \dot{\mathbf{y}})$ in (1) leads to a linear governing equation, referred to as the linear subsystem in the sequel.

In Eq. (1), the components of $\mathbf{f}(t)$ are supposed to be evolutionary processes. These random processes belong to the family of nonstationary processes, widely used in civil and mechanical engineering. Formally, the spectral distribution of the nodal forces is such that the vector of forces may be written in the form of a Fourier-Stieltjes integral, like

$\mathbf{f}(t)=\int_{\mathbb{R}} e^{i \omega t} \mathbf{a}(t, \omega) \mathrm{d} \tilde{\mathbf{f}}(\omega)$

with $\iota=\sqrt{-1}$ and $\mathbf{a}(t, \omega): \mathbb{R}^{+} \times \mathbb{R} \mapsto \mathbb{R}^{n \times n}$ being a diagonal matrix gathering deterministic time windows (also called intensity functions or time envelopes) and $\tilde{\mathbf{f}}(\omega, \theta): \mathbb{R} \times \Theta \mapsto \mathbb{C}^{n}$ being the vector of spectral processes related to the embedded stationary processes. This formulation is used by Priestley on the basis of the developments of Bartlett [2].

In a more particular case, but very often encountered in practice, all the stationary forces on a structure are modulated by the same time window $a(t, \omega)$. Most of the time, this assumption is used in seismic engineering. In wind engineering, the use of a matrix $\mathbf{a}(t, \omega)$ allows to model wind direction evolution during nonstationary storms.

Assuming $\mathbf{f}(t)$ a zero-mean process, the time-dependent covariance function of $\mathbf{f}(t)$, noted $\boldsymbol{\Sigma}_{\mathbf{f}}(t)$, is given by

$\boldsymbol{\Sigma}_{\mathbf{f}}(t)=\int_{\mathbb{R}} \hat{\mathbf{S}}_{\mathbf{f}}(t, \omega) \mathrm{d} \omega$

with $\hat{\mathbf{S}}_{\mathbf{f}}(t, \omega): \mathbb{R}^{+} \times \mathbb{R} \mapsto \mathbb{C}^{n \times n}$ being the evolutionary PSD of $\mathbf{f}(t)$. The hat used to denote evolutionary PSD stems from [39] to avoid any possible confusion with the PSD of the embedded stationary processes $\mathbf{S}_{\mathbf{f}}(\omega)$. Assuming that $\tilde{\mathbf{f}}(\omega)$ is a random process with orthogonal increments [25,39], $\widehat{\mathbf{S}}_{\mathbf{f}}(t, \omega)$ is

$\hat{\mathbf{S}}_{\mathbf{f}}(t, \omega)=\mathbf{a}(t, \omega) \mathbf{S}_{\mathbf{f}}(\omega) \mathbf{a}^{T}(t, \omega)$.

This previous equation summarizes the general philosophy of the evolutionary spectral representation: an embedded stationary process mainly described by its cross-PSD matrix and a modulation matrix introducing the time dependency in the problem. For the sake of simplicity and clarity in the following developments, 
the matrix $\mathbf{a}(t, \omega)$ is supposed to have the same function $a(t, \omega)$ on all entries of the diagonal. It is not necessary for the evolutionary processes considered in this work to be of the separable kind, i.e. not necessary that $a(t, \omega)$ be expressed as a product of a function of $t$ and a function of $\omega$.

\subsection{Objectives of this work}

The main developments of this work aim at solving (1) provided $\mathbf{f}(t)$ is a vector of evolutionary processes, supposed to form a coherent random field. We propose to develop a semi-analytical approach to perform EvSp analysis, by advantageously exploiting the features of the frequency-domain method. In particular, linear EvSp analysis relies on the computation of the evolutionary transfer (EvTr) matrix, defined as the convolution integral between the (modal or nodal) impulse response (IR) matrix of the structure and the time window $a(t, \omega)$ [39]. Therefore, the application of this method requires to know the IR matrix and to efficiently perform this convolution integral.

For SDOF linear systems, the closed-form expression of the IR matrix is known [12] and the EvTr matrices can be estimated, provided the deterministic time window has a tractable expression $[13,27]$. For MDOF linear systems, these results can be applied if the equations of motion are decoupled after projection in a modal basis [28]. Each mode is thus considered as a SDOF system responding independently of the others and the IR matrix is diagonal. However, if the modes are mechanically coupled, as in the presence of non-proportional damping, the modal IR matrix ceases to be diagonal and no analytical expression is available. Numerical methods are thus necessary and its computation becomes so timeconsuming, that Monte Carlo simulations [63] are usually preferred in practical applications [39].

Our first proposition in this work is therefore to provide a closed-form approximation of the IR matrix for mechanically coupled systems. Based on a perturbation approach [32], previously investigated in $[16,5,6]$, we first compute an asymptotic expansion of the IR matrix. Then, classic calculus is used to find an asymptotic expansion of the EvTr matrix. In Section 2, an efficient and generalized recurrence relation is proposed in order to extend our developments to envelope functions with more intricate shapes.

In a second step, nonlinear forces are considered within the system and the central question thus pertains to the application of EvSp analysis in equivalent statistical linearization. Indeed, this method has not been effectively applied, mainly for computational reasons. In the meantime, the concept of evolutionary PSD has inspired the development of evolutionary wavelet spectrum for linear [42] and equivalent linear [62] dynamics. Some operations in EvSp analysis are actually implemented with difficulty. For instance, Kougioumtzoglou and Spanos [36] qualify the convolution integral between the IR matrix and the time window as intractable. However, these authors highlight the particular case for which the timescale of the time window and those of the system are clearly separated; the convolution integral can be drastically simplified, even avoided in some cases.

This approach, the so-called quasi-stationary [29], may still lead to inaccurate estimation and its theoretical bases might be considered as fallacious to some extent. Indeed, in equivalent statistical linearization, the separation of the timescales does not theoretically lead to this assumption, but actually to a multiple scales formulation of the problem [43], in which the equivalent properties evolve at a different timescale than those of the structure. Our second proposition in Section 3 is thus to extend the developments pursued for linear EvSp analysis to equivalent linearization. The multiple scales philosophy is a clever manner to show that the nonstationary equivalent system can be regarded as a linear time- variant (LTV) system highlighting multiple timescales. Finally, the method is illustrated on problems pertaining to civil engineering applications in Section 4.

\section{Linear evolutionary spectral analysis}

As a first step in this work, we just focus on the analysis of LTI structures, namely the linear subsystem in (1), subject to Gaussian loadings. We discuss the construction of the IR matrix for a large scale structure, mechanically coupled in its modal basis. The nodal displacements of the structure, gathered in $\mathbf{x}(t, \theta): \mathbb{R} \times \Theta \mapsto \mathbb{R}^{n}$, are thus Gaussian evolutionary processes.

\subsection{Analysis in the modal basis and discussion on existing shortcomings}

The structural response of a given LTI structure is advantageously computed using a limited number $m$ of linear normal modes with $m \ll n$ for large-dimensional structures. The matrix $\Phi$ is the $n \times m$-matrix collecting the normal modes resulting from the eigenproblem $\mathbf{K} \boldsymbol{\Phi}=\omega^{2} \mathbf{M \Phi}$. This basis is normalized to have unit generalized masses, such that

$\boldsymbol{\Phi}^{T} \mathbf{M} \boldsymbol{\Phi}=\mathbf{I}, \quad \boldsymbol{\Phi}^{T} \mathbf{K} \boldsymbol{\Phi}=\boldsymbol{\Omega}$,

with $\mathbf{I}$ being the $m \times m$-identity matrix and $\boldsymbol{\Omega}$ the generalized stiffness matrix.

Using the modal superposition principle [12], Eq. (1) reads

$\ddot{\mathbf{q}}+\mathbf{D} \dot{\mathbf{q}}+\mathbf{\Omega q}=\mathbf{p}$,

with $\mathbf{q}(t) \quad(\mathbf{x}=\mathbf{\Phi q})$ being the vector of modal coordinates, $\mathbf{D}=\boldsymbol{\Phi}^{T} \mathbf{C} \boldsymbol{\Phi}$ and $\mathbf{p}(t)=\boldsymbol{\Phi}^{T} \mathbf{f}(t)$. Even though the number of variables has been reduced by the modal projection, the equations of motion are not decoupled, because $\mathbf{D}$ is not necessarily diagonal. Indeed, the hypothesis on classically damped systems, which has often been adopted in structural engineering, is not suitable to resolve, for example, fluid-structure interaction problems since the D matrix is influenced by interaction with the flow-induced forces. Moreover, if passive control forces are used to reduce vibration of a large structure, this hypothesis is very often violated when, for example, dash-pots are placed at selected locations of the system (as shown later in the applications). In these cases, the decoupling advantage offered by the modal projection is lost, since the modes are mechanically coupled.

In a stationary setting, the PSD matrix of the modal coordinates $\mathbf{S}_{\mathbf{q}}(\omega)$ is given by

$\mathbf{S}_{\mathbf{q}}(\omega)=\mathbf{H}(\omega) \mathbf{S}_{\mathbf{p}}(\omega) \mathbf{H}^{*}(\omega)$,

where the superscript $*$ denotes the hermitian operator and $\mathbf{H}(\omega): \mathbb{R} \mapsto \mathbb{C}^{m \times m}$ is the full modal transfer matrix of the linear structure, defined as

$\mathbf{H}(\omega)=\left(\boldsymbol{\Omega}-\omega^{2} \mathbf{I}+\imath \omega \mathbf{D}\right)^{-1}$.

From classic structural dynamics [12], we know that a transient response of a structure starting from rest is expressed as the convolution integral between the Impulse Response (IR) matrix of modal coordinates $\mathbf{h}(t): \mathbb{R}^{+} \mapsto \mathbb{R}^{m \times m}$, and a given realization $\tilde{\theta}$ of the generalized force vector, i.e.

$\mathbf{q}(t, \tilde{\theta})=\int_{t_{0}}^{t} \mathbf{h}(t-u) \mathbf{p}(u, \tilde{\theta}) \mathrm{d} u$,

with $t_{0}$ being the initial time. Hence, according to the FourierStieltjes representation of $\mathbf{p}(t)$, the convolution integral (9) may be rewritten as 
$\mathbf{q}(t, \tilde{\theta})=\int_{\mathbb{R}} \mathbf{G}\left(t, t_{0}, \omega\right) \mathrm{d} \tilde{\mathbf{p}}(\omega, \tilde{\theta})$

with $\tilde{\mathbf{p}}(\omega)=\boldsymbol{\Phi}^{T} \tilde{\mathbf{f}}(\omega)$ and $\mathbf{G}\left(t, t_{0}, \omega\right)$ being the Evolutionary Transfer (EvTr) matrix defined as

$\mathbf{G}\left(t, t_{0}, \omega\right)=\int_{t_{0}}^{t} a(u, \omega) \mathbf{h}(t-u) e^{i \omega u} \mathrm{~d} u$.

Defined as the convolution integral between the IR matrix $\mathbf{h}(t)$ and the time window, the EvTr matrix expresses the cumulative effects of the past intensities (of the embedded processes) on the present state. In EvSp analysis, the evolutionary PSD matrix of the modal coordinates $\hat{\mathbf{S}}_{\mathbf{q}}\left(t, t_{0}, \omega\right)$ is given by

$\hat{\mathbf{S}}_{\mathbf{q}}\left(t, t_{0}, \omega\right)=\mathbf{G}\left(t, t_{0}, \omega\right) \mathbf{S}_{\mathbf{p}}(\omega) \mathbf{G}^{*}\left(t, t_{0}, \omega\right)$.

The covariance matrix of the modal coordinates $\boldsymbol{\Sigma}_{\mathbf{q}}\left(t, t_{0}\right)$, which is time dependent, is given by integrating $\widehat{\mathbf{S}}_{\mathbf{q}}\left(t, t_{0}, \omega\right)$ with respect to $\omega \in \mathbb{R}$. The evolutionary formalism separately deals with the time and frequency domains. Furthermore, Eq. (12) is similar to the stationary approach (7), as the time $t$ in (12) might be considered as a parameter.

In the light of the previous equations, we may review some reasons explaining the difficulties to perform EvSp analysis. Actually, the computation of the IR matrix required in (11) is a timeconsuming operation. However, for perfectly decoupled systems, the matrix $\mathbf{h}(t)$ is diagonal and known in a closed-form, as it contains the IR function of each modal coordinate. For coupled systems, like non-classically damped structures, the decoupled approach as proposed by Lord Rayleigh [51] is definitely insufficient [41]. Therefore, numerical procedures must be used to adequately take into account the mechanical coupling in the modal basis. An approach could be to numerically solve the $m$-DOF system for $m$ different initial conditions [12], but this option is actually not preferred as exposed by Lin and Cai [39]. Indeed, they point out that "the time spent to determine the impulse response function is greater than what is required subsequently to calculate the evolutionary cross-spectra", a reason for rather using the Fourier relation valid for LTI systems between $\mathbf{h}(t)$ and $\mathbf{H}(\omega)$, i.e.

$\mathbf{h}(t)=\int_{\mathbb{R}} \mathbf{H}(\omega) e^{\imath \omega t} \mathrm{~d} \omega$.

Even though it is just a well-known inverse Fourier transform, this expression is not readily computed since it is repeated for each element of (8). Then, the computation of (11) is a heavy operation. Moreover, an efficient method to perform this integral should be able to deal with any time window, numerically or analytically known. Thus, we think that using a closed-form approximation for $\mathbf{h}(t)$ may advantageously circumvent the computations of (11) and (13).

In the following part of this section, we develop an analytical method to this end, for slightly to moderately coupled structures. Our method is based on an asymptotic expansion of the modal transfer matrix, already investigated in a stationary setting $[16,5,6]$. After circumventing this first difficulty, an efficient procedure is exposed in order to deal with any time window and to substantially improve the computation of the EvTr matrix by means of a recurrence relation.

\subsection{Asymptotic approximation of the impulse response matrix}

The efficient computation of (13) is the key to perform EvSp analysis. The governing equations in the modal basis (6) are slightly to moderately coupled from a mechanical point of view, since $\mathbf{D}$ is a full matrix. If the structure is slightly coupled in the basis $\boldsymbol{\Phi}$, we can develop $\mathbf{H}(\omega)$ in a series of terms, known in a closed-form and readily integrated.
In this work, we use the advantage of considering the coupling in the modal equations as a perturbation around a main decoupled system [16,5]. This main decoupled structure is obtained by discarding the out-of-diagonal elements of the modal damping matrix. Subsequently, we extract the terms creating the coupling within the system, by splitting $\mathbf{D}$ into two matrices

$\mathbf{D}=\mathbf{D}_{d}+\mathbf{D}_{0}$,

where $\mathbf{D}_{d}$ and $\mathbf{D}_{o}$ are built as $\mathbf{D}_{d}=\mathbf{D} \circ \mathbf{I}$ (diagonal elements) and $\mathbf{D}_{o}=\mathbf{D}-\mathbf{D}_{d}$ (out-of-diagonal elements) with the symbol $\circ$ denoting the Hadamard product. With these notations, the modal transfer matrix (8) becomes

$\mathbf{H}(\omega)=\left(\mathbf{J}_{d}+\mathbf{J}_{o}\right)^{-1}$,

where

$\mathbf{J}_{d}(\omega)=\boldsymbol{\Omega}-\omega^{2} \mathbf{I}+\imath \omega \mathbf{D}_{d}$ and $\mathbf{J}_{o}(\omega)=\imath \omega \mathbf{D}_{o}$

gather diagonal and out-of-diagonal elements, respectively. The subscript $d$ recalls that a given matrix is diagonal by construction. The modal transfer matrix of the virtually decoupled system is also introduced as $\mathbf{H}_{d}(\omega)=\mathbf{J}_{d}^{-1}$. It corresponds to the modal transfer matrix that would be obtained if the coupling was readily neglected, i.e. the leading order term of our expansion.

Upon some smallness conditions on the product $\mathbf{H}_{d} \mathbf{J}_{0}$, Eq. (15) may be approximated by

$\mathbf{H}_{N}(\omega)=\left(\mathbf{I}+\sum_{k=1}^{N}\left(-\mathbf{H}_{d} \mathbf{J}_{0}\right)^{k}\right) \mathbf{H}_{d}$,

with $N$ being a truncation order. Actually, the series $\mathbf{H}_{N}(\omega)$ converges to $\mathbf{H}(\omega)$ for $N \rightarrow \infty$, if the maximum spectral radius of $\mathbf{H}_{d} \mathbf{J}_{0}$ for all $\omega \in \mathbb{R}$, noted $\rho_{\mathbf{J}}$ in the following, is less than one (more details are available in [6]). This convergence criterion measures the diagonal dominance of the sum $\mathbf{J}_{d}+\mathbf{J}_{o}$ [34], i.e. the importance of the diagonal terms compared with the out-of-diagonal ones. Mechanically speaking, the concept of dominance ensures a moderate coupling within the system.

Hence, the matrix $\mathbf{h}(t)$ may be approximated by replacing $\mathbf{H}(\omega)$ in (13) by the truncated asymptotic expansion (17). Thus, the IR matrix $\mathbf{h}_{N}(t)$ may be interpreted as the IR matrix of a main decoupled system $\mathbf{h}_{d}(t)$ perturbed by higher-order correction terms $\Delta \mathbf{h}_{k}(t)$, i.e.

$\mathbf{h}_{N}(t)=\mathbf{h}_{d}(t)+\sum_{k=1}^{N}(-1)^{k} \Delta \mathbf{h}_{k}(t)$,

where the integral expressions of the terms of $\mathbf{h}_{N}(t)$ are

$\mathbf{h}_{d}(t)=\int_{\mathbb{R}} \mathbf{H}_{d} e^{i \omega t} \mathrm{~d} \omega$,

$\Delta \mathbf{h}_{k}(t)=\int_{\mathbb{R}}\left(\mathbf{H}_{d} \mathbf{J}_{0}\right)^{k} \mathbf{H}_{d} e^{\imath \omega t} \mathrm{~d} \omega$.

The linearity of the Fourier operator in (13) ensures that the series (18) converges to $\mathbf{h}(t)$ for $N \rightarrow \infty$.

At this step, a reasonable question would be to ask how these developments can help in performing integral (13), since replacing (13) by (18) is not prima facie a significant progress. Two main reasons justify our approach. First, the elements of $\mathbf{h}_{d}(t)$ are the IR functions of the main decoupled system, i.e. the $i$ th element on the diagonal is the IR function of the $i$ th mode of the structure. Thus, the leading-order term of the series (18) is unequivocally known in a closed-form. Moreover, the higher-order correction terms have no unexpected dependence on $\omega$, they are just products of the 
elements of $\mathbf{H}_{d}(\omega)$ besides $\omega$ itself (through $\mathbf{J}_{o}$ ). Therefore, the aforementioned doubt can be definitely raised by noticing that the poles of the functions (elements) in $\mathbf{H}_{d}(\omega)$ are readily extracted. Their multiplicity within the integrands of (19) is calculated as well. Then, Cauchy's theorem is invoked and we find closed-form expressions for the correction terms $\Delta \mathbf{h}_{k}(t)$. These explicit expressions are given and discussed in the following.

With the same methodology, a suitable asymptotic expansion of the EvTr matrix is found by introducing the expansion (18) for $\mathbf{h}_{N}(t)$ into (11). Thus, the $N$-order asymptotic expansion of $\mathbf{G}\left(t, t_{0}, \omega\right)$ is given by

$\mathbf{G}_{N}\left(t, t_{0}, \omega\right)=\mathbf{G}_{d}\left(t, t_{0}, \omega\right)+\sum_{k=1}^{N}(-1)^{k} \Delta \mathbf{G}_{k}\left(t, t_{0}, \omega\right)$.

The leading order term of this expansion is a diagonal matrix containing the EvTr functions of the main decoupled system

$\mathbf{G}_{d}\left(t, t_{0}, \omega\right)=\int_{t_{0}}^{t} a(u, \omega) \mathbf{h}_{d}(t-u) e^{i \omega u} \mathrm{~d} u$,

while the higher-order correction terms are obtained by the convolution integral between the time window and the correction terms of the IR matrix

$\Delta \mathbf{G}_{k}\left(t, t_{0}, \omega\right)=\int_{t_{0}}^{t} a(u, \omega) \Delta \mathbf{h}_{k}(t-u) e^{\imath \omega u} \mathrm{~d} u$.

For slightly to moderately coupled structures, Eq. (21) constitutes the core of the method developed for LTI systems. The proposed developments avoid the numerical computation of the inverse Fourier transform to get the impulse response matrix of the coupled system. They also avoid the repeated inversion of matrices in (8). For usual time envelopes, closed-form expressions can be found for the leading order and the correction terms. To the knowledge of the authors, this method can be ranked among the most efficient approaches to deal with coupling in EvSp analysis, since an important part of the computation is analytically treated.

Here the philosophy of this work is clearly presented. By applying the asymptotic expansion-based method, we are able to go further in the mathematical understanding of the equation of motion and to find reliable results for the solution of classic problems in engineering. In the following, some examples will illustrate this purpose and the validity of the proposed approach, but some mathematical results are first provided to clarify the computations of (18) and (21).

\subsection{Closed-form expression for the evolutionary transfer matrix}

Only keeping the leading order in the expansion (21), the $i$ th IR function related to the $i$ th structural mode reads

$\left(\mathbf{h}_{d}(t)\right)_{i}=\frac{e^{\Omega_{i}^{+} t}-e^{\Omega_{i}^{-} t}}{2 \imath \omega_{i} \sqrt{1-\xi_{i}^{2}}}$

with

$\Omega_{i}^{ \pm}=-\xi_{i} \omega_{i} \pm \imath \omega_{i} \sqrt{1-\xi_{i}^{2}}$,

$(\boldsymbol{\Omega})_{i}=\omega_{i}^{2}$ and $\left(\mathbf{D}_{d}\right)_{i}=2 \xi_{i} \omega_{i}$ for $i=1, \ldots m$. Introducing (24) into

(22), the $i$ th diagonal element of $\mathbf{G}_{d}\left(t_{1}, t_{0}, \omega\right)$ is given by

$\left(\mathbf{G}_{d}\left(t_{1}, t_{0}, \omega\right)\right)_{i}=\frac{\mathcal{I}_{i}^{+}\left(t_{1}, t_{0}, \omega\right)-\mathcal{I}_{i}^{-}\left(t_{1}, t_{0}, \omega\right)}{2 \iota \omega_{i} \sqrt{1-\xi_{i}^{2}}}$

with the integral $\mathcal{I}_{k}^{\alpha}\left(t_{1}, t_{0}, \omega\right)$ with $\alpha=\{+,-\}$, defined as

$I_{k}^{\alpha}\left(t_{1}, t_{0}, \omega\right)=e^{\Omega_{k}^{\alpha} t_{1}} \int_{t_{0}}^{t_{1}} a(u, \omega) e^{\left(\omega \omega-\Omega_{k}^{\alpha}\right) u} \mathrm{~d} u$.
For slightly coupled systems, the computation of the decoupled approximation offers already a good insight into the system behavior, especially about the covariance matrix of $\mathbf{q}(t)$. For moderately coupled systems, the computation of higher-order correction terms is mandatory. The first correction term of the EvTr matrix reads

$\Delta \mathbf{G}_{1}\left(t_{1}, t_{0}, \omega\right)=\int_{t_{0}}^{t_{1}} a(u, \omega) \Delta \mathbf{h}_{1}\left(t_{1}-u\right) e^{i \omega u} \mathrm{~d} u$.

The $\Delta \mathbf{h}_{1}(u)$ matrix is first computed by setting $k=1$ in (20). After some algebra, this matrix is conveniently rewritten, as

$\Delta \mathbf{h}_{1}(u)=\mathbf{D}_{o} \bigcirc \delta \mathbf{h}_{I}(u)$,

with $i, j=1, \ldots m$ and

$\left(\delta \mathbf{h}_{I}(u)\right)_{i j}=\int_{\mathbb{R}} \iota \omega\left(\mathbf{H}_{d}\right)_{i}\left(\mathbf{H}_{d}\right)_{j} e^{\imath \omega u} \mathrm{~d} \omega$.

The four poles of the integrand in (30) are $\iota \Omega_{i}^{ \pm}$and $\imath \Omega_{j}^{ \pm}$. Invoking Cauchy's theorem, Eq. (30) becomes

$$
\begin{aligned}
\left(\delta \mathbf{h}_{I}(u)\right)_{i j}= & \left(\mathbf{V}_{1}\right)_{i j} \exp \left(\Omega_{i}^{+} u\right)+\left(\mathbf{V}_{2}\right)_{i j} \exp \left(\Omega_{i}^{-} u\right)+\left(\mathbf{V}_{3}\right)_{i j} \exp \left(\Omega_{j}^{+} u\right) \\
& +\left(\mathbf{V}_{4}\right)_{i j} \exp \left(\Omega_{j}^{-} u\right)
\end{aligned}
$$

for $i \neq j$ [37]. The case of $i=j$ is particular, as the multiplicity of the poles is changed and Eq. (31) should be adapted. This case is not broached here, because $\mathbf{D}_{o}$ only has zero elements on its diagonal, so the computation of $\left(\delta \mathbf{h}_{I}\right)_{i i}$ is not required. The constant matrices $\mathbf{V}_{1}, \ldots \mathbf{V}_{4}$ read

$$
\begin{aligned}
\left(\mathbf{V}_{1}\right)_{i j}= & \frac{-l \Omega_{i}^{+}}{2 \omega_{i} \sqrt{1-\xi_{i}^{2}}\left(\Omega_{i}^{+}-\Omega_{j}^{-}\right)\left(\Omega_{i}^{+}-\Omega_{j}^{+}\right)}, \\
& \left(\mathbf{V}_{2}\right)_{i j}=\frac{\imath \Omega_{i}^{-}}{2 \omega_{i} \sqrt{1-\xi_{i}^{2}}\left(\Omega_{i}^{-}-\Omega_{j}^{-}\right)\left(\Omega_{i}^{-}-\Omega_{j}^{+}\right)}, \\
& \left(\mathbf{V}_{3}\right)_{i j}=\frac{-\imath \Omega_{j}^{+}}{2 \omega_{j} \sqrt{1-\xi_{j}^{2}}\left(\Omega_{j}^{+}-\Omega_{i}^{+}\right)\left(\Omega_{j}^{+}-\Omega_{i}^{-}\right)}, \\
& \left(\mathbf{V}_{4}\right)_{i j}=\frac{\imath \Omega_{j}^{-}}{2 \omega_{j} \sqrt{1-\xi_{j}^{2}}\left(\Omega_{j}^{-}-\Omega_{i}^{+}\right)\left(\Omega_{j}^{-}-\Omega_{i}^{-}\right)} .
\end{aligned}
$$

These constants only depend on the projection in the modal basis. For LTI systems, they are thus computed once and for all at the beginning of the analysis. The $m^{2}$ Fourier transforms in (13) are thus transformed, thanks to the asymptotic expansion-based method, into the sum (31), for which only the estimation of $4 \mathrm{~m}^{2}$ constants are required. However, the major computational gain of the method is found in the convolution integral (28).

Actually, the first-order correction term of the EvTr matrix $\mathbf{G}_{N}\left(t, t_{0}, \omega\right)$ now reads

$\Delta \mathbf{G}_{1}\left(t_{1}, t_{0}, \omega\right)=\mathbf{D}_{o} \bigcirc \delta \mathbf{G}_{I}\left(t_{1}, t_{0}, \omega\right)$,

since the system is time-invariant. The matrix $\delta \mathbf{G}_{I}$, defined as

$\delta \mathbf{G}_{I}\left(t_{1}, t_{0}, \omega\right)=\int_{t_{0}}^{t_{1}} a(u, \omega) \delta \mathbf{h}_{I}\left(t_{1}-u\right) e^{i \omega u} \mathrm{~d} u$,

is computed by using the previously defined integral $I_{i}^{\alpha}$. Indeed, substitution of (31) into (34) leads to

$\left(\delta \mathbf{G}_{I}\left(t_{1}, t_{0}, \omega\right)\right)_{i j}=\left(\mathbf{V}_{1}\right)_{i j} \mathcal{I}_{i}^{+}+\left(\mathbf{V}_{2}\right)_{i j} \mathcal{I}_{i}^{-}+\left(\mathbf{V}_{3}\right)_{i j} \mathcal{I}_{j}^{+}+\left(\mathbf{V}_{4}\right)_{i j} \mathcal{I}_{j}^{-}$.

The computation of this first correction term is rapid. It does not demand any more complicated operation than the computation of the time-invariant matrices $\mathbf{V}_{1}, \ldots \mathbf{V}_{4}$ and the integrals $\mathcal{I}_{i}^{\alpha}$.

Again, the underlying philosophy of the perturbation approach developed in Section 2.2 is clearly highlighted. Applications of 
analytical methods and linear algebra through the asymptotic expansion-based method allow to avoid some heavy computational operations. The understanding of the governing equations and the identification of general properties in structures lead to develop mathematical tools in order to extract blocks of elementary results. Although numerical sampling methods can always be applied for those kinds of problems, the main advantage in our semi-analytical approach is to simplify them into more basic problems. Thence, we contradict the notorious idea that a transient problem can only be efficiently solved by simulations.

The implementation flowchart given as Algorithm 1 summarizes the different steps of the analysis, with this proposed method.

Algorithm 1. Evolutionary analysis of a linear system with windowing function $a(t)$ having a simple analytical expression (i.e. (27) is explicit).

1: Set up equation of motion, determine mode shapes and frequencies of undamped system, split $\mathbf{D}$ into $\mathbf{D}_{d}$ and $\mathbf{D}_{0}$, see (14)

2: Compute and store the PSD matrix of modal forces $\mathbf{S}_{\mathbf{p}}=\boldsymbol{\Phi}^{T} \mathbf{S}_{\mathbf{f}} \Phi$

3: Choose $\Delta t, t_{\text {end }}$

4: Choose $\Delta \omega, \omega_{\text {end }}$

$t_{1} \leftarrow t_{0}+\Delta t, \Sigma_{q}\left(t_{1}, t_{0}\right) \leftarrow 0$

while $t_{1}<t_{\text {end }}$ do

while $\omega<\omega_{\text {end }}$ do

Recall $\mathbf{S}_{\mathbf{p}}(\omega)$

Determine $I_{k}^{\alpha}\left(t_{1}, t_{0}, \omega\right)$

Determine the diagonal matrix $\mathbf{G}_{d}$

Determine successive corrections $\delta \mathbf{G}_{1}, \ldots \delta \mathbf{G}_{N}$

Construct $\mathbf{G}_{N}=\mathbf{G}_{d}+\delta \mathbf{G}_{1}+\cdots+\delta \mathbf{G}_{N}$

$\boldsymbol{\Sigma}_{q}\left(t_{1}, t_{0}\right) \leftarrow \boldsymbol{\Sigma}_{q}\left(t_{1}, t_{0}\right)+\mathbf{G}_{N} \mathbf{S}_{\mathbf{p}} \mathbf{G}_{N}^{*} \Delta \omega$

$\omega \leftarrow \omega+\Delta \omega$

end while

$t_{1} \leftarrow t_{1}+\Delta t$

: end while

The computation of $\mathcal{I}_{i}^{\alpha}$, defined on the interval $\left[t_{0}, t_{1}\right]$ in (27), depends on the shape of the time envelope $a(t, \omega)$. The time window in (27) must be sufficiently simple to have closed-form expression of these integrals. For instance, this integral is readily performed for the Shinozuka window [58], widely used in earthquake engineering. Nevertheless, this is not necessarily the case in practice, if we consider the Jenning window in earthquake engineering or the Holmes window for downburst in wind engineering [33]. The generalization of our developments to any time window is described in the next section.

\subsection{Convenient recurrence procedure}

We propose to rewrite the modal equation of motion (6) into a state space formalism

$\dot{\mathbf{z}}=\mathbf{A z}+\mathbf{B}\left(\begin{array}{l}\mathbf{0} \\ \mathbf{p}\end{array}\right)$

with $\mathbf{A}$ and $\mathbf{B}$ being two $2 m$-dimensional matrices, constant in time, and $\mathbf{z}(t, \theta): \mathbb{R}^{+} \times \Theta \mapsto \mathbb{R}^{2 m}$ the modal state vector of the system, all defined as

$\mathbf{A}=\left(\begin{array}{cc}\mathbf{0} & \mathbf{I} \\ -\boldsymbol{\Omega} & -\mathbf{D}\end{array}\right), \quad \mathbf{B}=\left(\begin{array}{ll}\mathbf{0} & \mathbf{0} \\ \mathbf{0} & \mathbf{I}\end{array}\right) \quad$ and $\quad \mathbf{z}=\left(\begin{array}{l}\mathbf{q} \\ \dot{\mathbf{q}}\end{array}\right)$

The general solution of LTI systems is expressed as

$\mathbf{z}(t, \tilde{\theta})=\Psi\left(t, t_{0}\right) \mathbf{z}\left(t_{0}\right)+\int_{t_{0}}^{t} \Psi(t, u) \mathbf{B}\left(\begin{array}{c}\mathbf{0} \\ \mathbf{p}(u, \tilde{\theta})\end{array}\right) \mathrm{d} u$

with $\Psi\left(t, t_{0}\right): \mathbb{R}^{+} \times \mathbb{R}^{+} \mapsto \mathbb{R}^{2 m \times 2 m}$ being the state transition matrix of the system in the modal basis from a time $t_{0}$ to a time $t$ and $\mathbf{z}\left(t_{0}\right)$ the initial condition $\left(t>t_{0}\right)$. In the following, we assume that $\mathbf{z}\left(t_{0}\right)=\mathbf{0}$. Introducing in (38) the Fourier-Stieltjes representation of $\mathbf{p}(t)$ (as in (2)), we can highlight a state space evolutionary transfer (StSpEvTr) matrix $\mathbf{r}\left(t, t_{0}, \omega\right): \mathbb{R}^{+} \times \mathbb{R}^{+} \times \mathbb{R} \mapsto \mathbb{C}^{2 m \times 2 m}$, defined as

$\mathbf{r}\left(t, t_{0}, \omega\right)=\int_{t_{0}}^{t} a(u, \omega) \Psi(t, u) \mathbf{B} e^{i \omega u} \mathrm{~d} u$

With the formalism used in (12), the evolutionary PSD $\widehat{\mathbf{S}}_{\mathbf{z}}\left(t, t_{0}, \omega\right)$ for the modal state vector $\mathbf{z}(t)$ is given by

$\hat{\mathbf{S}}_{\mathbf{z}}\left(t, t_{0}, \omega\right)=\mathbf{r}\left(t, t_{0}, \omega\right)\left(\begin{array}{cc}\mathbf{0} & \mathbf{0} \\ \mathbf{0} & \mathbf{S}_{\mathbf{p}}(\omega)\end{array}\right) \mathbf{r} *\left(t, t_{0}, \omega\right)$.

The time-dependent covariance matrix $\boldsymbol{\Sigma}_{\mathbf{z}}\left(t, t_{0}\right)$ is found by integrating $\hat{\mathbf{S}}_{\mathbf{z}}\left(t, t_{0}, \omega\right)$ with respect to $\omega \in \mathbb{R}$. With this formalism, the correlation between displacements and velocities, which is not null in a nonstationary setting, is immediately computed.

At this step, a relation between the state space formalism and the asymptotic expansion-based method may be emphasized, since the state transition matrix $\Psi\left(t_{1}, t_{0}\right)$ can be related to the impulse response matrix $\mathbf{h}(t)$ [31]. Indeed, the product between $\boldsymbol{\Psi}\left(t_{1}, t_{0}\right)$ and $\mathbf{B}$ is given by

$\boldsymbol{\Psi}\left(t_{1}, t_{0}\right) \mathbf{B}=\left(\begin{array}{cc}\mathbf{0} & \mathbf{h}\left(t_{1}-t_{0}\right) \\ \mathbf{0} & \partial_{t_{1}} \mathbf{h}\left(t_{1}-t_{0}\right)\end{array}\right)$,

since the properties of the structure are time-independent [31]. Thence, the StSpEvTr matrix $\mathbf{r}\left(t, t_{0}, \omega\right)$ is obtained by substituting (41) into (39) and by invoking the definition (11) of $\mathbf{G}\left(t, t_{0}, \omega\right)$, such that

$\mathbf{r}\left(t, t_{0}, \omega\right)=\left(\begin{array}{cc}\mathbf{0} & \mathbf{G}\left(t, t_{0}, \omega\right) \\ \mathbf{0} & \partial_{t} \mathbf{G}\left(t, t_{0}, \omega\right)\end{array}\right)$

with $\mathbf{h}(0)=\mathbf{0}$. The state space formalism introduced in (36) is therefore related to the modal coordinates in (6). By replacing $\mathbf{G}\left(t, t_{0}, \omega\right)$ by its asymptotic expansion (21), we obtain the $N$-order asymptotic expansion of $\mathbf{r}\left(t, t_{0}, \omega\right)$, noted $\mathbf{r}_{N}\left(t, t_{0}, \omega\right)$ in the following.

The state space formalism is more advantageous than the modal space formalism to compute the convolution integrals hidden in (42), as it naturally considers the correlation between displacements and velocities, extremely important during transient motion regimes. Based on this, we now provide an interesting recurrence strategy to compute $\mathbf{r}\left(t, t_{0}, \omega\right)$ on the whole interval $\left[t_{0}, t\right]$, without computing the integrals $\mathcal{I}_{i}^{\alpha}$ on this interval.

First, the whole time interval is divided into $M+1$ non-overlapping intervals, such as

$t_{0}=0<\cdots<t_{k}<\cdots t_{M} \leq t \leq t_{M+1}$,

with $k, M \in \mathbb{N}_{0}$ and with possibly different step lengths. Assuming piecewise continuity of the functions involved in the StSpEvTr matrix $\mathbf{r}\left(t, t_{0}, \omega\right)$, Eq. (39) may be rewritten as

$$
\begin{aligned}
\mathbf{r}\left(t, t_{0}, \omega\right)= & \sum_{k=1}^{M} \int_{t_{k-1}}^{t_{k}} a(u, \omega) \Psi(t, u) \mathbf{B} e^{i \omega u} \mathrm{~d} u+\int_{t_{M}}^{t} a(u, \omega) \Psi(t, u) \\
& \mathbf{B} e^{\imath \omega u} \mathrm{~d} u .
\end{aligned}
$$

The well-known semi-group property of the transition matrix $\Psi\left(t, t_{0}\right)[31]$ can now be used. It expresses that the transition between two instants $t_{0}$ and $t$ is equivalent to a first transition from $t_{0}$ to an intermediate time $t_{1}$, then a second transition from $t_{1}$ to $t$, i.e.

$\boldsymbol{\Psi}\left(t, t_{0}\right)=\boldsymbol{\Psi}\left(t, t_{1}\right) \mathbf{\Psi}\left(t_{1}, t_{0}\right)$.

This useful property, coming from the state space formalism, allows to make the computation of integrals in (44) independent of the final time $t$, such that 


$$
\begin{aligned}
\mathbf{Y}\left(t, t_{0}, \omega\right)= & \sum_{k=1}^{M} \boldsymbol{\Psi}\left(t, t_{k}\right) \int_{t_{k-1}}^{t_{k}} a(u, \omega) \Psi\left(t_{k}, u\right) \\
& \mathbf{B} e^{\imath \omega u} \mathrm{~d} u+\int_{t_{M}}^{t} a(u, \omega) \Psi(t, u) \mathbf{B} e^{\omega \omega u} \mathrm{~d} u
\end{aligned}
$$

In this equation, we identify the last term as $\mathbf{r}\left(t, t_{M}, \omega\right)$, i.e. the StSpEvTr matrix on $\left[t_{M}, t\right]$. This matrix may be approximated by its $\mathrm{N}$-order asymptotic expansion, such as

$\mathbf{r}_{N}\left(t, t_{M}, \omega\right)=\left(\begin{array}{cc}\mathbf{0} & \mathbf{G}_{N}\left(t, t_{M}, \omega\right) \\ \mathbf{0} & \partial_{t} \mathbf{G}_{N}\left(t, t_{M}, \omega\right)\end{array}\right)$.

Then, we use the semi-group property between the three instants $t, t_{M}$ and $t_{k}$, i.e. $\boldsymbol{\Psi}\left(t, t_{k}\right)=\boldsymbol{\Psi}\left(t, t_{M}\right) \Psi\left(t_{M}, t_{k}\right)$, in the summation (46). Therefore, we find a convenient recurrence relation for the computation of $\mathbf{r}\left(t, t_{0}, \omega\right)$, approximated by $\mathbf{r}_{N}\left(t, t_{0}, \omega\right)$, i.e.

$\mathbf{r}_{N}\left(t, t_{0}, \omega\right)=\boldsymbol{\Psi}\left(t, t_{M}\right) \mathbf{r}_{N}\left(t_{M}, t_{0}, \omega\right)+\mathbf{r}_{N}\left(t, t_{M}, \omega\right)$.

Eq. (48) is of paramount importance in this work and the physical meaning of the two terms is noteworthy. The first term expresses that the past of the stochastic system up to time $t_{M}$, summarized in the function $\mathbf{r}\left(t_{M}, t_{0}, \omega\right)$, is directly projected to time $t$ by the transition matrix between these two instants. The second term models the linear response of the system for the loads appearing during the interval $\left[t_{M}, t\right]$.

From a computational point of view, this relation is really efficient for three main reasons.

- The state transition matrix $\boldsymbol{\Psi}\left(t, t_{M}\right)$, equal to the exponential matrix

$\Psi\left(t, t_{M}\right)=e^{\left(t-t_{M}\right) \mathbf{A}}$,

is efficiently computed by a Padé approximation, as implemented in MatLab. Moreover, it is not involved in any convolution integral and only depends on the interval length $\left|t-t_{M}\right|$.

- The asymptotic approximation is not involved in the computation of $\boldsymbol{\Psi}\left(t, t_{M}\right)$. Therefore, the coupling is formally taken into account in the projection of $\mathbf{r}_{N}\left(t_{M}, t_{0}, \omega\right)$ to time $t$. The coupling within the system is just approximated for the computation of $\mathbf{r}_{N}\left(t, t_{M}, \omega\right)$. Actually, it is shown in the next section that the shorter the time interval $\left[t_{M}, t\right]$, the less correction terms are required to converge to $\mathbf{r}\left(t, t_{M}, \omega\right)$.

- The transition from $t_{M}$ to $t$ only requires to compute the convolution integral over the time interval $\left[t_{M}, t\right]$, and not over the whole time domain $\left[t_{0}, t\right]$ as in (39). Therefore, this recurrence relation enables to use any time window $a(t, \omega)$, numerically known or with intractable analytical shape. Indeed, the intensity of the time window may be assumed, locally, to evolve linearly between two instants. The window is thus replaced by a piecewise linear approximation and the convolution in $\mathbf{G}_{N}\left(t, t_{M}, \omega\right)$ is readily computed with the antiderivative

$\mathcal{P}_{k, j}^{ \pm}=\int u^{j} e^{-W_{k}^{ \pm} u} \mathrm{~d} u=\frac{e^{-\mathcal{W}_{k}^{ \pm} u}}{-\mathcal{W}_{k}^{ \pm}} u^{j}+\frac{j}{\mathcal{W}_{k}^{ \pm}} \mathcal{P}_{k, j-1}^{ \pm}$

with $W_{k}^{ \pm}=\Omega_{k}^{ \pm}-\imath \omega$.

\subsection{About the convergence of the asymptotic expansions}

The conditions upon which the asymptotic expansions derived above shall be valid are now discussed. Concerning the asymptotic expansion $\mathbf{H}_{N}(\omega)$, the series is convergent if the convergence criterion $\rho_{\mathbf{J}}$ is less than one. The index $\rho_{\mathbf{J}}$ is the maximum spectral radius of $\mathbf{H}_{d} \mathbf{J}_{0}$ for all $\omega \in \mathbb{R}$, i.e.
$\rho_{\mathbf{J}}=\max _{\omega \in \mathbb{R}}\left\{\Gamma\left[\mathbf{H}_{d}(\omega) \mathbf{J}_{o}(\omega)\right]\right\}<1$,

with $\Gamma[\mathbf{U}]=\max \left\{\left|\lambda_{\mathbf{U}, i}\right|, i=1, \ldots m\right\}$ being the spectral radius of $\mathbf{U} \in \mathbb{C}^{m \times m}$ and $\lambda_{\mathbf{U}, i}$ the $i$ th eigenvalue of $\mathbf{U}$ [34]. The maximum maximorum in (51) is found for $\omega$ in a neighborhood of $\omega_{i}$, a natural frequency of the structure, as shown by Canor et al. in $[6,4]$. The convergence criterion $\rho_{\mathbf{J}}$ is a key indicator in this part. It must be calculated in each application to check the validity of the expansion.

If the series $\mathbf{H}_{N}(\omega)$ converges, the convergence of the series $\mathbf{h}_{N}(t)$ is also ensured by the linearity of the Fourier operator. However, the rate of convergence is not necessarily preserved. Actually, the criterion $\rho_{\mathbf{J}}$ applied to $\mathbf{h}_{N}(t)$ is a convergence criterion in the long term, since $\mathbf{H}_{N}(\omega)$ is related to the stationary response of the structure in the frequency domain. The matrix $\mathbf{h}_{N}(t)$ features a transient regime before this stationary response. Numerical experiments have shown that the required number of correction terms $N$ is reduced for a given accuracy, if short time intervals are considered, compared with the stationary solution $(t \rightarrow \infty)$.

For the sake of clarity, we propose to illustrate this convergence property in the light of a particular case where $\mathbf{H}_{d}(\omega)=\bar{H}(\omega) \mathbf{I}$ in (19) with

$\bar{H}(\omega)=\frac{1}{\left(\omega_{0}^{2}-\omega^{2}\right)+\left(2 \imath \xi \omega_{0} \omega\right)}$.

Thence, we assume that all the modes have the same natural frequency and the same damping ratio. The integral (19) is now rewritten as

$\Delta \mathbf{h}_{k}(t)=\int_{\mathbb{R}}(l \omega)^{k} \bar{H}(\omega)^{k+1} \mathbf{D}_{o}^{k} e^{l \omega t} \mathrm{~d} \omega=\bar{h}_{k}(t) \mathbf{D}_{o}^{k}$

for $k \in \mathbb{N}$. Since we are mainly interested in the functions $\bar{h}_{k}(t)$ around $t=0$, we only compute the leading order term of their Taylor expansion, which reads

$\bar{h}_{k}(t)=\frac{2 \pi t^{k+1}}{k+1 !}+O\left(t^{k+2}\right)$.

Provided the time $t$ is small, the correction given by $\bar{h}_{k}$ becomes negligible. This short demonstration explains, heuristically, the reason why the number of required terms in $\mathbf{h}_{N}(t)$ is reduced on short time intervals. This important property allows us to consider less correction terms in $\mathbf{h}_{N}$ than in $\mathbf{H}_{N}(\omega)$. However, the importance of the criterion $\rho_{\mathbf{J}}<1$ is not modified, nor weakened, since it must be satisfied in any case. In light of this property, the convergence of the expansions with our approach is more conveniently verified by increasing the number of intervals $M$ instead of increasing the number of correction terms $N$. The following examples show that $N=2$ provides already very accurate results.

To summarize the analysis procedure with the modal statespace representation of the structure, an implementation flowchart is given in Algorithm 2.

Algorithm 2. Evolutionary analysis of a linear system - general state-space formulation - valid for any time window $a(t)$.

1: Set up equation of motion, determine mode shapes and frequencies of undamped system. Construct matrix A, see (37)

2: Compute and store the PSD matrix of modal forces $\mathbf{S}_{\mathbf{p}}=\boldsymbol{\Phi}^{T} \mathbf{S}_{\mathbf{f}} \Phi$

3: Choose $\Delta t$, $t_{\text {end }}$

4: Choose $\Delta \omega, \omega_{\text {end }}$

$\checkmark$ time marching

5: $\quad t_{M} \leftarrow t_{0}, t \leftarrow t_{M}+\Delta t, \Sigma_{z}\left(t, t_{0}\right) \leftarrow 0$,

D numerical Integration over

$\mathbf{Y}_{N}\left(t_{0}, t_{0}, \omega\right) \leftarrow 0$

6: while $t<t_{\text {end }}$ do

7: $\quad$ while $\omega<\omega_{\text {end }}$ do $\triangleright$ initialize

8: $\quad$ Recall $\mathbf{S}_{\mathbf{p}(\omega)}$ repeat until $t_{\text {end }}$ - integrate on $\omega \in\left[0, \omega_{\text {end }}\right]$ 


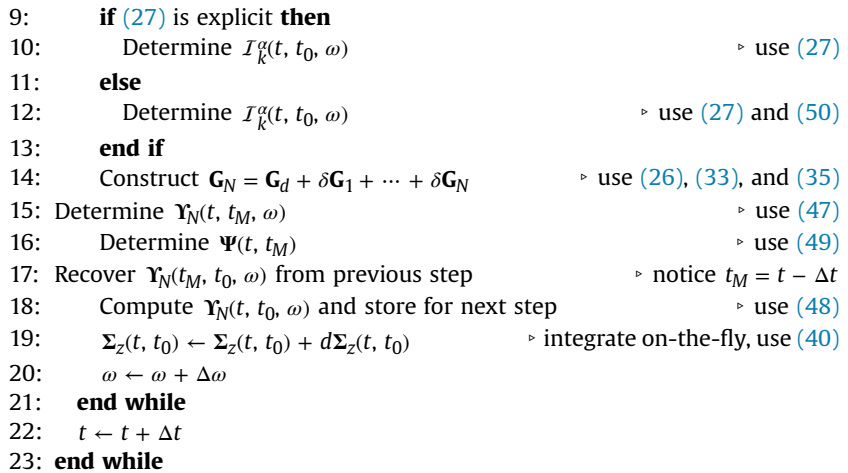

\section{Nonlinear evolutionary spectral analysis}

\subsection{Gaussian equivalent linearization}

The results of the previous section have been obtained in the context of linear EvSp analysis. The scope of this section is now to extend the asymptotic expansion-based method, as well as the generalized procedure, to nonlinear dynamics by means of the GEL.

The equivalent statistical linearization, originally introduced by Botoon and Caughey [7,8] can be used for the analysis of largescale nonlinear structures, as encountered in earthquake engineering $[14,69,22]$ or in wind engineering $[71,26,24]$. The main idea of the equivalent linearization consists in replacing the nonlinear system with an equivalent linear one by minimizing an error criterion depending on the parameters of the equivalent system. The adjective Gaussian highlights that the random responses are assumed to be Gaussian processes.

The stochastic linearization consists in replacing Eq. (1) by the equation of motion of a $n$-DOF equivalent linear system, which reads

$\mathbf{M} \ddot{\mathbf{x}}+\left(\mathbf{C}+\mathbf{C}_{e q}(t)\right) \dot{\mathbf{x}}+\left(\mathbf{K}+\mathbf{K}_{e q}(t)\right) \mathbf{x}=\mathbf{f}$,

where $\mathbf{x}(t, \theta): \mathbb{R}^{+} \times \Theta \mapsto \mathbb{R}^{n}$ gathers the Gaussian nodal displacements of the equivalent linear system and where $\mathbf{K}_{e q}$ and $\mathbf{C}_{e q}$ are the equivalent stiffness and damping matrices, respectively [52]. These equivalent matrices are determined by minimizing the error function $\mathcal{E}(t)$ [25], defined as

$\mathcal{E}(t)=E\left[\mathbf{E}^{T} \mathbf{E}\right], \quad \mathbf{E}(t)=\mathbf{K}_{e q} \mathbf{x}+\mathbf{C}_{e q} \dot{\mathbf{x}}-\mathbf{g}(\mathbf{x}, \dot{\mathbf{x}})$

with $E[$. ] being the expectation operator. For usual nonlinear mappings $\mathbf{g}$, the expectations in the previous equation may be expressed as a function of the covariance matrix of the response, as

$\mathbf{K}_{e q}(t)=\mathcal{K}\left(\mathbf{\Sigma}_{\mathbf{x} \mathbf{x}}, \mathbf{\Sigma}_{\dot{\mathbf{x}} \dot{\mathbf{x}}}, \mathbf{\Sigma}_{\mathbf{x} \dot{\mathbf{x}}}\right)$,

$\mathbf{C}_{e q}(t)=C\left(\boldsymbol{\Sigma}_{\mathbf{x} \mathbf{x}}, \boldsymbol{\Sigma}_{\dot{\mathbf{x}} \mathbf{x}}, \boldsymbol{\Sigma}_{\mathbf{x} \mathbf{x}}\right)$

with $\mathcal{K}$ and $C$ being two operators depending on the nonlinear behaviors modeled in $\mathbf{g}$.

If the equivalent stiffness matrix $\mathbf{K}_{e q}$ is small compared with the stiffness matrix $\mathbf{K}$, the projection of (55) into the basis $\boldsymbol{\Phi}$ of the linear normal modes remains suitable to represent the actual deformed configuration. This strong assumption prevents this approach to be implemented in the case of strong nonlinearities where the equivalent stiffness matrix may significantly differ from the stiffness of the original linear system. With this assumption,
Eq. (55) reads

$\ddot{\mathbf{q}}+\left(\mathbf{D}+\mathbf{D}_{e q}(t)\right) \dot{\mathbf{q}}+\left(\boldsymbol{\Omega}+\boldsymbol{\Omega}_{e q}(t)\right) \mathbf{q}=\mathbf{p}(t)$,

with $\boldsymbol{\Omega}_{e q}=\boldsymbol{\Phi}^{T} \mathbf{K}_{e q} \boldsymbol{\Phi}$ and $\mathbf{D}_{e q}=\boldsymbol{\Phi}^{T} \mathbf{C}_{e q} \boldsymbol{\Phi}$. As previously, the equations of motion are not decoupled, as $\mathbf{D}_{e q}$ and $\boldsymbol{\Omega}_{e q}$ are not necessarily diagonal. Therefore, the uncoupling advantage offered by the modal projection is lost. Moreover, we shall notice that Eq. (59) characterizes a linear time-variant (LTV) system now. The timedependence is not explicit, the equivalent matrices in (57) depend upon time, because of the time evolution of the covariance matrices.

As in (36), the equation of motion (59) may be rewritten in a state space formalism,

$\dot{\mathbf{z}}=\left(\begin{array}{cc}\mathbf{0} & \mathbf{I} \\ -\left(\boldsymbol{\Omega}+\boldsymbol{\Omega}_{e q}\right) & -\left(\mathbf{D}+\mathbf{D}_{e q}\right)\end{array}\right) \mathbf{z}+\mathbf{B}\left(\begin{array}{l}\mathbf{0} \\ \mathbf{p}\end{array}\right) \equiv \mathbf{A}_{e q}(t) \mathbf{z}+\mathbf{B}\left(\begin{array}{l}\mathbf{0} \\ \mathbf{p}\end{array}\right)$

with $\mathbf{A}_{e q}(t)$ being the equivalent convection matrix, readily identified as the equivalent statistical extension of (37). As the equivalent system belongs to the broad family of LTV systems, the general solution of (60) may be expressed as

$\mathbf{z}(t)=\int_{t_{0}}^{t} \Psi_{e q}(t, u)\left(\begin{array}{c}\mathbf{0} \\ \mathbf{p}(u)\end{array}\right) \mathrm{d} u$,

with $\Psi_{e q}\left(t, t_{0}\right)$ being the equivalent state transition matrix from $t_{0}$ to $t$. This equation is identical to (38) with the noteworthy difference that this matrix is here defined for the equivalent system. As done for LTI systems, an equivalent StSpEvTr matrix $\mathbf{\Upsilon}_{e q}\left(t, t_{0}, \omega\right): \mathbb{R}^{+} \times \mathbb{R}^{+} \times \mathbb{R} \mapsto \mathbb{C}^{2 m \times 2 m}$ (with $t>t_{0}$ ) may be defined as

$\mathbf{\Upsilon}_{e q}\left(t, t_{0}, \omega\right)=\int_{t_{0}}^{t} a(u, \omega) e^{i \omega u} \Psi_{e q}(t, u) \mathbf{B d} u$.

Some developments carried out in the previous section could be applied in this case, but the Fourier relation (13) ceases to be valid for LTV systems. Furthermore, Eq. (49) cannot be applied, since $\mathbf{A}_{e q}$ is time-dependent. This issue may however be circumvented by taking advantage of the major specificity of this equivalent LTV system. Indeed, there are different timescales in the problem: the statistical timescale and the structural timescale. This noteworthy fact is theoretically formalized in the following with a multiple timescales approach. It will allow to interpret the equivalent LTV system as a piecewise LTI system evolving according to a slow statistical timescale.

\subsection{Multiple scales approach in stochastic linearization}

The time evolution of the vector of modal coordinates $\mathbf{q}(t)$ may be modeled by the linearized modal equations of motion

$\ddot{\mathbf{q}}+\left(\mathbf{D}+\mathbf{D}_{e q}(t)\right) \dot{\mathbf{q}}+\left(\boldsymbol{\Omega}+\boldsymbol{\Omega}_{e q}(t)\right) \mathbf{q}=a(t) \mathbf{w}(t)$

with $a(t)$ being a deterministic time envelope and $\mathbf{w}(t, \theta): \mathbb{R}^{+} \times \Theta \mapsto \mathbb{R}^{m}$ a random excitation vector, supposed to gather $\delta$-correlated processes. Although this vector may be possibly colored in order to model more realistic excitations, we decide to consider white noises in this demonstration for the sake of simplicity.

After recasting (63) into a state space formalism, the time evolution of the covariance matrix $\boldsymbol{\Sigma}_{\mathbf{z}}$ is described with a Lyapunov equation by invoking Itô's lemma [47], such that

$\dot{\boldsymbol{\Sigma}}_{\mathbf{z}}=\mathbf{A}_{e q}(t) \boldsymbol{\Sigma}_{\mathbf{z}}+\boldsymbol{\Sigma}_{\mathbf{z}} \mathbf{A}_{e q}^{T}(t)+a(t)^{2}\left(\begin{array}{cc}\mathbf{0} & \mathbf{0} \\ \mathbf{0} & \mathbf{S}_{\mathbf{W}}\end{array}\right)$

with $\mathbf{S}_{\mathbf{W}}$ gathering the white noise intensities. Eq. (64) is a firstorder ordinary differential equation, while Eq. (63) is a second- 
order one. No oscillations are thus expected in the time evolution of $\mathbf{\Sigma}_{\mathbf{z}}(t)$ contrary to $\mathbf{q}(t)$ or $\mathbf{z}(t)$. Thence, the equations of motion reflect different behaviors characterized by different timescales and this Lyapunov equation reflects the existence per se of a statistical timescale, slower than the structural one.

The application of the multiple scales technique [32,43], widely used for decades in mechanics $[40,21,57,17]$, and in random dynamics [53,3], looks perfectly appropriate, since an analysis of the different regimes is mandatory at this step. A convenient way to sort the timescales is to introduce a distinction between the fast timescale $t_{f}$ and the slow timescale $t_{s}$. The fast one is related to high frequency contents, i.e. to both the system natural periods and the frequency content of the excitation. The slow one is the statistical timescale related to the deterministic envelope and the memory time of the system, since they both condition the time evolution of the response statistics.

Now, a small parameter $\varepsilon$ is introduced to compare the time variables $t_{f}$ and $t_{s}$ with the physical time $t$, such that

$\left\{\begin{array}{c}t_{f}=t \\ t_{s}=\varepsilon t\end{array}\right.$

With the multiple scales approach, the dependence on time $t\left(t_{f}, t_{s}\right)$ may be seen as a function of $t_{s}$ and/or $t_{f}$ in accordance with the underlying phenomenon. Thus, the ordinary differential operator in time becomes the partial differential operator

$\frac{\mathrm{d} \cdot}{\mathrm{d} t}=\frac{\partial \cdot}{\partial t_{f}}+\varepsilon \frac{\partial \cdot}{\partial t_{s}}$.

The modal equation of motion (63) reads

$$
\begin{aligned}
& \frac{\partial^{2} \mathbf{q}}{\partial t_{f}^{2}}+2 \varepsilon \frac{\partial^{2} \mathbf{q}}{\partial t_{f} \partial t_{s}}+\varepsilon^{2} \frac{\partial^{2} \mathbf{q}}{\partial t_{s}^{2}}+\left(\mathbf{D}+\mathbf{D}_{e q}\right)\left(\frac{\partial \mathbf{q}}{\partial t_{f}}+\varepsilon \frac{\partial \mathbf{q}}{\partial t_{s}}\right)+\left(\boldsymbol{\Omega}+\boldsymbol{\Omega}_{e q}\right) \mathbf{q} \\
& \quad=a\left(t_{s}\right)\left(\mathbf{w}\left(t_{f}\right)+\varepsilon \mathbf{w}\left(t_{s}\right)\right)
\end{aligned}
$$

Setting $a(t)$ dependent only on $t_{s}$ (and not on $t_{f}$ ) is inspired by the physics of the phenomenon, i.e. a slowly varying envelope. The white noises in $\mathbf{w}(t)$ are split into two different terms by invoking the fractal property of the Wiener process [25]. For instance, the duration of an earthquake is usually longer than the fundamental period of a structure; this is even more true for a downburst of a thunderstorm.

In a classical multiple scales approach, the random vector $\mathbf{q}(t)$ becomes a function of both $t_{f}$ and $t_{s}$. A Poincaré expansion of $\mathbf{q}\left(t_{f}, t_{s}\right)$,

$\mathbf{q}\left(t_{f}, t_{s}\right)=\mathbf{q}_{0}\left(t_{f}, t_{s}\right)+\varepsilon \mathbf{q}_{1}\left(t_{f}, t_{s}\right)+\varepsilon^{2} \mathbf{q}_{2}\left(t_{f}, t_{s}\right)+\cdots$

is introduced in (67). The leading order equation, i.e. the equation extracted from the terms of order $\varepsilon^{0}=1$, is given by

$\frac{\partial^{2} \mathbf{q}_{0}}{\partial t_{f}^{2}}+\left(\mathbf{D}+\mathbf{D}_{e q}\left(\boldsymbol{\Sigma}_{\mathbf{z}}\right)\right) \frac{\partial \mathbf{q}_{0}}{\partial t_{f}}+\left(\boldsymbol{\Omega}+\boldsymbol{\Omega}_{e q}\left(\boldsymbol{\Sigma}_{\mathbf{z}}\right)\right) \mathbf{q}_{0}=a\left(t_{s}\right) \mathbf{w}\left(t_{f}\right)$.

From Eq. (69), we clearly notice that the dynamical response of the system is governed by the fast timescale.

We however show that the covariance matrix $\boldsymbol{\Sigma}_{\mathbf{z}}\left(t_{f}, t_{s}\right)$ mainly evolves according to the slow timescale $t_{s}$. To do so, we modify the role of $\varepsilon$ compared with (65): the fast timescale is stretched to be of the same order of magnitude as the slow one, i.e.

$\left\{\begin{array}{c}t_{f}=t / \varepsilon \\ t_{s}=t\end{array}\right.$

The ratio between $t_{f}$ and $t_{s}$ is preserved in both (65) and (70). This artifact is a key principle of the method, since it allows us to separately focus on the fast or the slow dynamics. This technique is actually used to analyze distinct asymptotic behaviors in a same problem.

The differential operator (66) now reads

$\frac{\mathrm{d} \cdot}{\mathrm{d} t}=\frac{1}{\varepsilon} \frac{\partial \cdot}{\partial t_{f}}+\frac{\partial \cdot}{\partial t_{s}}$

and the Lyapunov equation (64), governing the time evolution of $\Sigma_{\mathbf{z}}$, yields

$\frac{\partial \boldsymbol{\Sigma}_{\mathbf{z}}}{\partial t_{f}}+\varepsilon \frac{\partial \boldsymbol{\Sigma}_{\mathbf{z}}}{\partial t_{s}}=\varepsilon \mathbf{A}_{e q} \boldsymbol{\Sigma}_{\mathbf{z}}+\varepsilon \boldsymbol{\Sigma}_{\mathbf{z}} \mathbf{A}_{e q}^{T}+\varepsilon\left(\begin{array}{cc}0 & 0 \\ 0 & a\left(t_{s}\right)^{2} \mathbf{S}_{\mathbf{w}}\end{array}\right)$

with the term coming from the excitation only depending on $t_{s}$. The $\varepsilon$-asymptotic expansion of the covariance matrix also reads

$\boldsymbol{\Sigma}_{\mathbf{z}}\left(t_{f}, t_{s}\right)=\boldsymbol{\Sigma}_{\mathbf{z}, 0}\left(t_{f}, t_{s}\right)+\varepsilon \boldsymbol{\Sigma}_{\mathbf{z}, 1}\left(t_{f}, t_{s}\right)+\varepsilon^{2} \boldsymbol{\Sigma}_{\mathbf{z}, 2}\left(t_{f}, t_{s}\right)+\cdots$

and is introduced in (72). The leading order equation is given by

$\frac{\partial \mathbf{\Sigma}_{\mathbf{z}, 0}}{\partial t_{f}}=\mathbf{0}$.

This key result shows that, at leading order, the matrix $\boldsymbol{\Sigma}_{\mathbf{z}}$ only depends upon the slow timescale $t_{s}$ and so are the equivalent matrices. The leading order of (63) thus reads

$$
\begin{gathered}
\frac{\partial^{2} \mathbf{q}_{0}}{\partial t_{f}^{2}}+\left(\mathbf{D}+\mathbf{D}_{e q}\left(\boldsymbol{\Sigma}_{\mathbf{z}, 0}\left(t_{s}\right)\right)\right) \frac{\partial \mathbf{q}_{0}}{\partial t_{f}}+\left(\boldsymbol{\Omega}+\boldsymbol{\Omega}_{e q}\left(\boldsymbol{\Sigma}_{\mathbf{z}, 0}\left(t_{s}\right)\right)\right) \mathbf{q}_{0}=a\left(t_{s}\right) \\
\mathbf{w}\left(t_{f}\right),
\end{gathered}
$$

taking into account the physical separation between the fast timescale and the statistical one. The separation of the time-scales between the structure and the statistical response leads to consider the statistical LTV system as piecewise LTI system, since the equivalent properties evolve more slowly than the structural response. This concept is formalized in the following.

\subsection{Piecewise approximation of the equivalent system}

According to the multiple scales developments, the equivalent damping and stiffness matrices evolve with the statistical time, i.e. the slow timescale of the stochastic system. Based on this fact, the interval $\left[t_{0}, t\right]$ can be divided with regard to the length of envelope and the memory time of the system. Actually, for over-damped structures, the memory time is ranked among the fast timescale. The continuous representation of the equivalent matrices can be approximated by a piecewise constant function. For instance, the equivalent convection matrix reads

$\mathbf{A}_{e q}(t) \approx \sum_{k=1}^{M} \mathbf{A}_{e q}\left(\tilde{t}_{k}\right) u\left(t \in\left[t_{k-1}, t_{k}\right)\right)$

with $\tilde{t}_{k} \in\left[t_{k-1}, t_{k}\right)(k=1, \ldots M)$ and $\square(\mathcal{A})$ being the indicator function of a set $\mathcal{A}$. The same approximation is applied to $\mathbf{C}_{e q}(t)$ and $\mathbf{K}_{e q}(t)$.

In (43), the total time interval $\left[t_{0}, t\right]$ is divided into $M+1$ nonoverlapping intervals. Since the properties of the system are supposed to be constant on the $k$ th time interval $\left[t_{k-1}, t_{k}\right)$, the equivalent state transition matrix $\Psi_{e q, k}\left(t_{2}, t_{1}\right)$ on this interval is given by

$\Psi_{e q, k}\left(t_{2}, t_{1}\right)=e^{\mathbf{A}_{e q}\left(\tilde{t}_{k}\right)\left(t_{2}-t_{1}\right)}$

with $t_{1}, t_{2}, \tilde{t}_{k} \in\left[t_{k-1}, t_{k}\right)$. The semi-group property of the transition matrices $\Psi_{e q, k}\left(t_{2}, t_{1}\right)$ applies to the piecewise approximation, as well as the developments exposed in Section 2.4.

Mutatis mutandis, the equivalent StSpEvTr matrix reads 
$\mathbf{r}_{e q}\left(t, t_{0}, \omega\right)=\Psi_{e q, M}\left(t, t_{M-1}\right) \mathbf{r}_{e q}\left(t_{M-1}, t_{0}, \omega\right)+\mathbf{Y}_{e q}\left(t, t_{M-1}, \omega\right)$,

where

$\mathbf{Y}_{e q}\left(t, t_{M-1}, \omega\right)=\left(\begin{array}{cc}\mathbf{0} & \mathbf{G}_{e q}\left(t, t_{M-1}, \omega\right) \\ \mathbf{0} & \partial_{t} \mathbf{G}_{e q}\left(t, t_{M-1}, \omega\right)\end{array}\right)$

and $t \in\left[t_{M-1}, t_{M}\right)$. Moreover, $\mathbf{G}_{e q}\left(t, t_{k-1}, \omega\right)$ with $t \in\left[t_{k-1}, t_{k}\right)$ is the equivalent EvTr matrix on the interval $\left[t_{k-1}, t_{k}\right)$ with the equivalent properties constant on this interval. Eq. (78), similar to (48), but in the context of equivalent statistical linearization, is a fundamental result of this work. Thanks to the multiple scales approach, we demonstrate that the efficient formulation developed in Section 2.4 can be used in equivalent statistical linearization in a nonstationary setting. The main difference is that the equivalent matrices are modified on each interval.

The convolution integral leading to $\mathbf{G}_{e q}\left(t, t_{M-1}, \omega\right)$ is performed at a negligible computational cost by the proposed asymptotic expansion-based method. Initially developed in a stationary setting, then extended to linear evolutionary analysis, it is now definitely applicable to nonstationary equivalent statistical linearization. Therefore, we readily replace $\mathbf{G}_{e q}$ by $\mathbf{G}_{e q, N}$ in (79) and follow the same implementation flowchart as for the linear structural behavior, see Algorithm 2.

There is however a notable difference in the nonlinear case. If the modal basis results from the eigenproblem $\mathbf{K \Phi}=\omega^{2} \mathbf{M \Phi}$, the matrix $\boldsymbol{\Omega}_{e q}$ is certainly non-diagonal. The sum $\boldsymbol{\Omega}+\boldsymbol{\Omega}_{e q}$ is thus split into $\boldsymbol{\Omega}_{d}+\boldsymbol{\Omega}_{0}$, i.e. a diagonal (d) matrix and an off-diagonal (o) one, as well as the damping matrices $\mathbf{D}+\mathbf{D}_{e q}=\mathbf{D}_{d}+\mathbf{D}_{0}$. The matrices defined in (16) become $\mathbf{J}_{d}=\boldsymbol{\Omega}_{d}-\omega^{2} \mathbf{I}+\imath \omega \mathbf{D}_{d}$ and $\mathbf{J}_{o}=\boldsymbol{\Omega}_{o}+\imath \omega \mathbf{D}_{0}$. The analytical expressions of the correction terms with both $\boldsymbol{\Omega}_{o}$ and $\mathbf{D}_{0}$ are a bit more involved; they can be found in [4], since we only consider $\mathbf{D}_{o}$ in Section 2.2. Nonetheless, a small criterion $\rho_{\mathbf{I}}$ ensures that the modal basis $\boldsymbol{\Phi}$ is adequate for the problem and that the matrix $\mathbf{K}_{e q}$ is small compared with $\mathbf{K}$ in $\boldsymbol{\Phi}$.

Some comments are now given about the time step in the piecewise approximation. The a priori division of the time interval $\left[t_{0}, t\right]$ depends on the different timescales characterizing the system. For highly to moderately damped structures with short memory time, the statistics of the response are mainly governed by the time window which must not be restricted to the whole duration of the action. For instance, the aforementioned three phases of an earthquake must be considered individually. A natural period less than a phase of the time envelope allows us to consider larger time steps in the resolution. Nonetheless, the time interval used for the piecewise approximation of the equivalent matrices may be of the order of the fast timescale $t_{f}$, since evolutions of the structure, well-estimated on large time intervals, are identically captured on smaller ones. Moreover, the convergence properties are such that the refinement of the division of $\left[t_{0}, t\right]$ do not necessarily lead to a better result.

Finally, the time $\tilde{t}_{k}$ inside the $k$ th interval $\left[t_{k-1}, t_{k}\right.$ ) can be conveniently chosen as the lower bound of the interval. The discussion on this choice pertains to a balance between the computational burden and the accuracy of the implementation. Since no information about the property on the $k$ th interval is available at $t_{\mathrm{k}-1}$, a suitable approximation of $\mathbf{A}_{e q}\left(\tilde{t}_{k}\right)$ in (77) is computed with the covariance matrix $\boldsymbol{\Sigma}_{\mathbf{z}}$ at the end of the interval $\left[t_{k-2}, t_{k-1}\right)$. Moreover, this explicit scheme favors the use of short time steps, which are well appreciated for the convergence of the asymptotic expansion.

\section{Illustrations}

\subsection{Applications in earthquake engineering}

An application in earthquake engineering inspired by [45] is now proposed. The evolutionary spectral analysis is used to compute the response of a multi-span bridge subject to an earthquake, modeled as a coherent random field $[35,72,1]$.

\subsubsection{Structure subject to differential ground acceleration}

In the presence of differential seismic excitation, the vector of nodal displacement $\mathbf{x}(t)$ is split into two vectors: $\mathbf{x}_{S}(t)$ containing the free DOFs of the structure and $\mathbf{u}_{g}$ the fixed ones at the foundations. The equation of motion [1] thus reads

$\left(\begin{array}{cc}\mathbf{M} & \mathbf{M}_{c} \\ \mathbf{M}_{c}^{T} & \mathbf{M}_{g}\end{array}\right)\left(\begin{array}{l}\ddot{\mathbf{x}}_{S} \\ \ddot{\mathbf{u}}_{g}\end{array}\right)+\left(\begin{array}{cc}\mathbf{C} & \mathbf{C}_{c} \\ \mathbf{C}_{c}^{T} & \mathbf{C}_{g}\end{array}\right)\left(\begin{array}{c}\dot{\mathbf{x}}_{S} \\ \dot{\mathbf{u}}_{g}\end{array}\right)+\left(\begin{array}{cc}\mathbf{K} & \mathbf{K}_{c} \\ \mathbf{K} s_{c}^{T} & \mathbf{K}_{g}\end{array}\right)\left(\begin{array}{c}\mathbf{x}_{S} \\ \mathbf{u}_{g}\end{array}\right)=\left(\begin{array}{c}0 \\ \mathbf{f}_{g}\end{array}\right)$

with $\mathbf{M}, \mathbf{M}_{c}$ and $\mathbf{M}_{g}$ being the blocks of the global mass matrix corresponding to degrees-of-freedom $s$ and $g$. The same division is applied to the damping and the stiffness matrices. The vector $\mathbf{f}_{g}(t)$ gathers the reaction forces induced by the random accelerations $\ddot{\mathbf{u}}_{g}(t)$ at the ground level. The first line of $(80)$ is recast into

$\mathbf{M} \ddot{\mathbf{x}}_{S}+\mathbf{C} \dot{\mathbf{x}}_{S}+\mathbf{K} \mathbf{x}_{S}=-\mathbf{M}_{c} \ddot{\mathbf{u}}_{g}-\mathbf{C}_{c} \dot{\mathbf{u}}_{g}-\mathbf{K}_{c} \mathbf{u}_{g}$.

The vector $\mathbf{x}_{S}(t)$ is decomposed into a pseudo-static component $\mathbf{x}_{B}$ and a dynamic one $\mathbf{x}_{R}$, such that

$\mathbf{x}_{S}=\mathbf{x}_{B}+\mathbf{x}_{R}$

with $\mathbf{x}_{B}=\mathbf{R} \mathbf{u}_{g}$ and $\mathbf{R}=-\mathbf{K}^{-1} \mathbf{K}_{c}$. Substitution of (82) into (81) yields

$\mathbf{M} \ddot{\mathbf{x}}_{R}+\mathbf{C} \dot{\mathbf{x}}_{R}+\mathbf{K} \mathbf{x}_{R}=-\left(\mathbf{M R}+\mathbf{M}_{c}\right) \ddot{\mathbf{u}}_{g}$,

since the contribution to the excitation coming from the damping forces may be neglected [30]. The projection of (83) into the modal basis $\Phi$ reads

$\ddot{\mathbf{q}}_{R}+\mathbf{D} \dot{\mathbf{q}}_{R}+\mathbf{\Omega} \mathbf{q}_{R}=-\boldsymbol{\Phi}^{T}\left(\mathbf{M R}+\mathbf{M}_{c}\right) \ddot{\mathbf{u}}_{g}$

with the notation similar to (6) and with $\mathbf{x}_{R}=\mathbf{\Phi} \mathbf{q}_{R}$.

The spectral characteristics of $\ddot{\mathbf{u}}_{g}$ are now given. In earthquake engineering, the concept of wave transportation leads to consider complex-valued coherence function. The PSD matrix of ground acceleration may be modeled by

$\left(\mathbf{S}_{\dot{\mathbf{u}}}(\omega)\right)_{i j}=S_{\ddot{u}}(\omega)\left|\Gamma\left(\omega, d_{i j}\right)\right| \exp \left(-l \frac{\omega d_{i j}}{V}\right)$

with $S_{u i}(\omega)$ being the unilateral psd of ground acceleration (e.g. the Kanai-Tajimi spectrum), $d_{i j}$ the distance between the points $i$ and $j$ and $V$ the apparent wave velocity (between 1 and $4 \mathrm{~km} / \mathrm{s}$ ). The complex exponential in (85) models the wave passage effect, while the norm of $\Gamma\left(\omega, d_{i j}\right)$, usually referred to as the lagged coherency [72] or the incoherency [18]. In earthquake engineering, suitable and general expressions for coherence function are obtained with difficulty, since dense installation of instruments are required to compensate for the scarcity of the recorded data. Here (as in [45]), we use the expression proposed by Hariachandran and Vanmarcke [30] based on data recorded throughout the SMART-1 project,

$\left|\Gamma\left(\omega, d_{i j}\right)\right|=A \exp \left(\frac{d_{i j} \chi_{A}}{\delta \vartheta(\omega)}\right)+(1-A) \exp \left(\frac{d_{i j} \chi_{A}}{\vartheta(\omega)}\right)$

with 
$\vartheta(\omega)=k_{\vartheta}\left(1+\left|\frac{\omega}{\omega_{\vartheta}}\right|^{b}\right)^{-\frac{1}{2}}$,

$\chi_{A}=-2(1-A+\delta A), A=0.736, \quad \delta=0.147, \quad \omega_{\vartheta}=6.85 \mathrm{rad} / \mathrm{s}$ and $b=2.78$. The length $k_{\vartheta}$ is ranged from 5 to $10 \mathrm{~km}$ to adapt the coherence function.

\subsubsection{Characteristics of the multi-span bridge}

The bridge, considered in this example and illustrated in Fig. 1, counts 20 spans of $40 \mathrm{~m}$ long and the piles are $20 \mathrm{~m}$ high. The mechanical properties of the deck are $E=40 \mathrm{GPa}, I=40 \mathrm{~m}^{4}$, $A=6 \mathrm{~m}^{2}$ and those of the piles are $E=60 \mathrm{GPa}, I=9 \mathrm{~m}^{4}, A=14 \mathrm{~m}^{2}$. The density is 3 tons $/ \mathrm{m}^{3}$ for deck and pile elements.

At the pile-deck connections on piles 4,10 and 16, damping devices are set up in order to mitigate seismic vibration. The behavior of these dampers is modeled by the classic velocity-force law

$F_{D}(v)=C_{D} \operatorname{sign}(\mathrm{v})|\mathrm{v}|^{\alpha}$

with $v$ being the relative velocity between the top of the pile and the deck and $C_{D}$ the damping coefficient. The exponent $\alpha$ is generally less than one in order to mitigate vibration at low velocity. Based on all these structural characteristics, a FE model is built up with 5 elements for each pile and each span. The damping matrix of the structure without dampers is constructed by setting the damping ratio equal to $1.0 \%$ in the first and third modes. The first five natural frequencies of this structure are 1.28, 1.29, 1.30, 1.33 and $1.40 \mathrm{~Hz}$. The shape of the first mode is also shown in Fig. 1.

Concerning the excitation, the parameters of the modified Kanai-Tajimi [12,6] spectrum $S_{u ̈}$ (in (85)) are $S_{0}=3 \cdot 10^{-3} \mathrm{~m}^{2} \mathrm{~s}^{-3}$, $\omega_{1}=2.5 \pi \mathrm{rad} / \mathrm{s}, \quad \xi_{1}=0.2, \quad \omega_{2}=0.5 \mathrm{rad} / \mathrm{s}, \quad \xi_{2}=0.6$ and Jenning's window is considered

$a(t)= \begin{cases}\left(t / t_{1}\right)^{2}, & 0<t \leq t_{1} \\ 1, & t_{1}<t \leq t_{2} \\ \exp \left(\gamma\left(t_{2}-t\right)\right) & t_{2} \leq t\end{cases}$

with $t_{1}=3 \mathrm{~s}, t_{2}=10 \mathrm{~s}$ and $\gamma=1 \mathrm{~Hz}$.

\subsubsection{Results and comparisons}

In this application, the analysis is performed according to the approach exposed in Section 3. The time window of $20 \mathrm{~s}$ is sampled with a $0.5 \mathrm{~s}$ time step $(M=40)$ and the cross-psd of the excitation is sampled with 5000 points essentially gathered around the natural frequencies. The results are validated by comparison with 10,000 digital simulations computed with a time step of $\Delta t=2.4 \mathrm{~ms}$. The embedded stationary process is generated with a frequency resolution of $\Delta f=3 \mathrm{~m} \mathrm{~Hz}$.

In Fig. 2, the convergence of the truncated series is first investigated for the linear case $(\alpha=1)$ with $C_{D}=20 \mathrm{MNs} / \mathrm{m}$ and so $\rho_{\mathbf{J}}=0.74$. For this index, the modal responses obtained with the decoupled approximation $(N=0)$ are not apparently accurate enough in comparison with the responses obtained with $N=2$. However, the asymptotic expansion converges quite fast, in spite of a quite large index of convergence, since the results obtained with $N=1$, not shown in Fig. 2, are perfectly superimposed on those obtained with $N=2$. This fact is previously highlighted in Section 3, where we emphasize that the convergence of the series $\mathbf{h}_{N}(t)$ is improved on shorter time intervals compared with the convergence of $\mathbf{H}_{N}(\omega)$. To deeper investigate this different rate of convergence, we compare the stationary response computed with the expansion (17) against the asymptotic approach in EvSp analysis. We set $\gamma=0$ in Jenning's window in order to evolve to the steady-state response.

In Fig. 3, the reference stationary response $(N=\infty)$ and the stationary decoupled approximation are depicted at $t=30 \mathrm{~s}$ with the markers $(\bullet)$ and $(\square)$, respectively. The curves show the evolution to the steady state for $N=0$ and $N=2$. The comparison between the curves and the markers explicitly illustrates our purpose. The transient decoupled approximation (dotted line) is much closer to the target response $(\bullet)$ than the stationary decoupled one $(\square)$, while the transient coupled approximation $N=2$ meets the target response. We attribute these different behaviors to the proposed efficient formulation.

Actually, the state transition matrix $\Psi\left(t, t_{M-1}\right)$ is not frequency dependent and can be numerically computed taking into account the modal coupling. Therefore, the asymptotic approximation of the modal coupling is only considered on the interval $\left[t_{M-1}, t\right]$. Furthermore, provided this interval is not too long compared with the characteristic times of the structure, a single correction term is sufficient, since the rate of convergence is improved on short time steps.

After this example in linear dynamics, we propose to illustrate the multiple scales approach in nonlinear dynamics by considering three sets of parameters for the damping law: (i) $\alpha=0.75$,

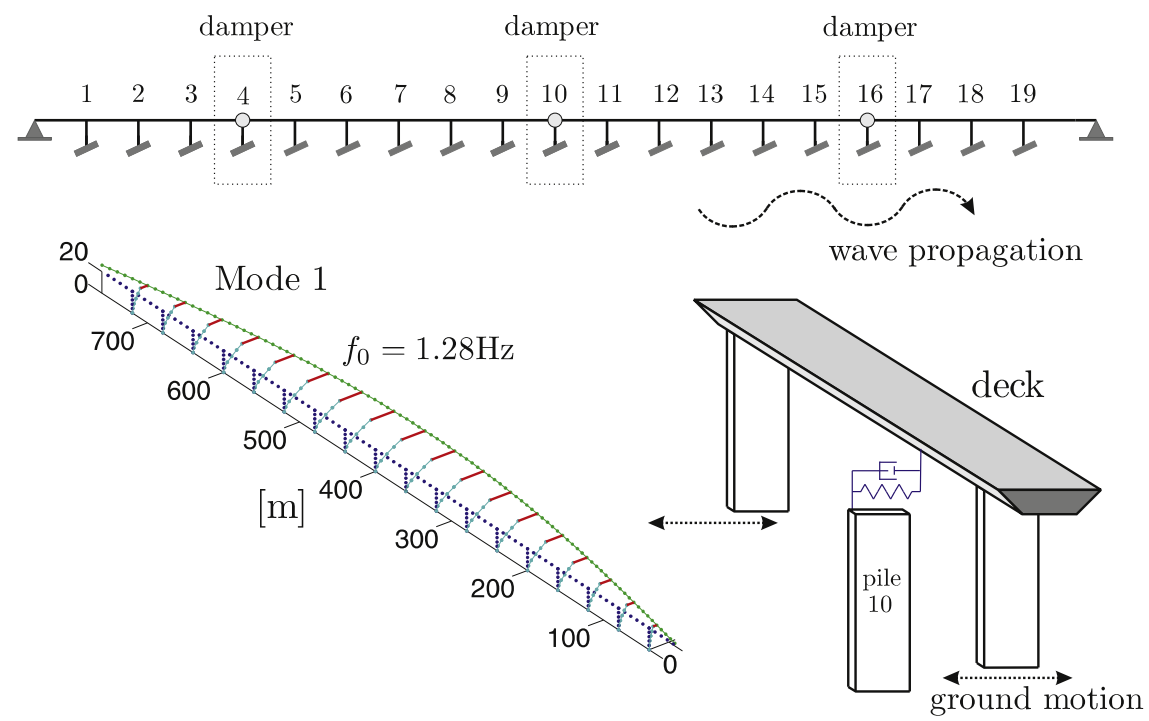

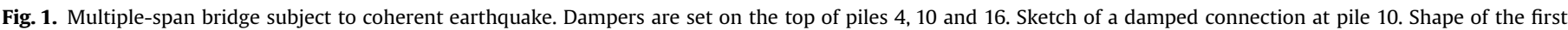
mode. 


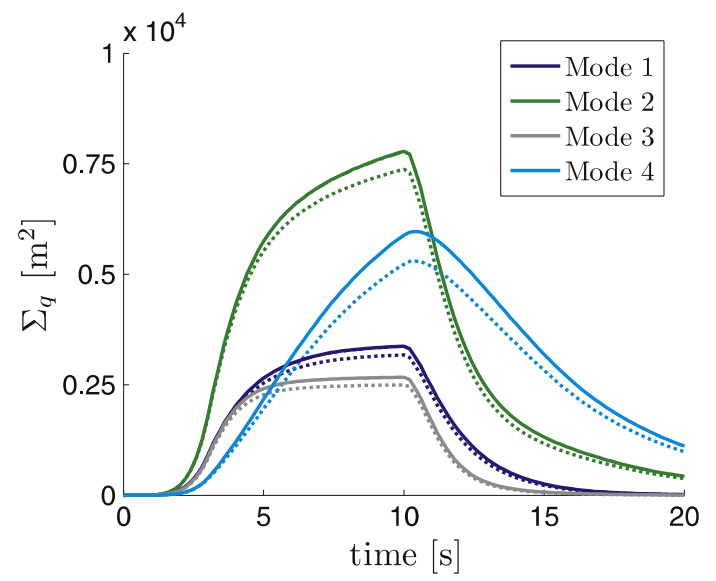

Fig. 2. Multi-span bridge under coherent earthquake. Variances of the modal coordinates. $C_{D}=20 \mathrm{MNs} / \mathrm{m}, \alpha=1$ and $\rho_{\mathrm{J}}=0.74$. Results obtained with $N=0$ (dotted line) and $N=2$ (continuous line). Results obtained with $N=1$ (not plotted here) and $N=2$ are superimposed.

$C_{D}=5 \cdot 10^{6} \mathrm{~N}(\mathrm{~s} / \mathrm{m})^{\alpha}, \quad$ (ii) $\quad \alpha=0.50, \quad C_{D}=3 \cdot 10^{6} \mathrm{~N}(\mathrm{~s} / \mathrm{m})^{\alpha}$ and (iii) $\alpha=0.25, C_{D}=10^{6} \mathrm{~N}(\mathrm{~s} / \mathrm{m})^{\alpha}$. However, this law is non-differentiable for $v=0$ and the initial increment of damping force, for a structure vibrating from rest, is thus infinite. The Kazakov formula [52] directly emphasizes an ill-conditioned linearization in a transient regime. To circumvent this difficulty, the nonlinear law (88) is replaced by a linear behavior in $v \in\left[0, v_{0}\right]$ with $v_{0}=0.01 \mathrm{~m} / \mathrm{s}$. This modification is considered in the Monte Carlo simulations as well. In the asymptotic approach, the truncation order $N$ is equal to 2 . Though the convergence of the results has been compared with $N=1$, it is not illustrated here. The comparison with Monte Carlo simulation ( $\Delta t=0.01 \mathrm{~s}, \Delta f=1 \mathrm{~m} \mathrm{~Hz}$ and 3200 samples) should be sufficient to assess the convergence for the concerned reader.

The main results obtained for this example are plotted in Fig. 4 for three different values of $\alpha$. In Figs. 4a and b, we notice the good agreement between our method and Monte Carlo simulations. In particular, the perturbation method follows very well the different phases of the transient response. In Fig. 4c, the results obtained with our method match less well the simulations, because of the higher intensity of the nonlinear forces. We do not forget that the equivalent statistical linearization is more accurate for weakly nonlinear forces. The dampers introduce a strong connection between the top of the piles and the deck. Fig. $4 \mathrm{~d}$ emphasizes that the convergence criterion $\rho_{\mathbf{J}}$ of the series is high, but mostly less

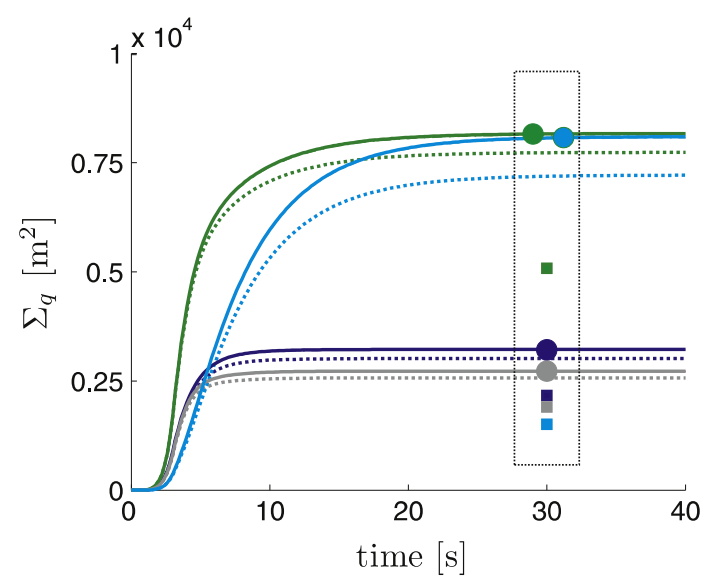

Fig. 3. Multi-span bridge under coherent earthquake. Variances of the modal coordinates, evolution to the steady state. $C_{D}=20 \mathrm{MNs} / \mathrm{m}, \alpha=1$ and $\rho_{\mathbf{J}}=0.74$. Results obtained with $N=0$ and $N=2$. The legend is similar to Fig. 2. Results from stationary spectral analysis shown with markers: $\boldsymbol{\Sigma}_{\mathbf{q}_{d}}(\square)$, i.e. decoupled approximation, and $\boldsymbol{\Sigma}_{\mathbf{q}, \infty}(\bullet)$. than unity throughout the solution.

\subsection{Applications in wind engineering}

In wind buffeting analysis, the wind velocity is usually assumed to be a stationary Gaussian process. However, some well-known phenomena in wind engineering fail to be physically modeled with the stationary assumption. A downburst, which is a notable example, is a sudden fall of a column of air sinking from the higher atmospheric level that spreads out in all directions after impinging the ground. According to [33], such an event normally occurs during thunderstorms. Some pertinent theories have been developed in the last decade to apply more realistic models of transient wind velocity $[68,11,56,73]$. For downbursts, Holmes et al. [33] propose an empirical model based on the translation velocity of the storm and the radial velocity of the air [10].

Holmes' model, illustrated in Fig. 5, compares an observation point $P$ to the trajectory of the storm supposed to move along a straight line $(y=0)$ with a constant translation velocity $V_{t}$. The offset distance between this line and the observation point is denoted as $\ell$. The center of the storm $O$, the so-called stagnation point, is located at a distance $V_{t} t$ from $Q$, the starting point of the storm [9]. At time $t$, the position vector $\mathbf{r}(t)=\overrightarrow{O P}$ is given by $\mathbf{r}=\left(d_{0}-V_{t} t, \ell\right)$. The radial velocity induced by the divergence of the air flux is given by

$\mathbf{V}_{r}=\frac{\mathbf{r}}{\|\mathbf{r}\|} V_{r}(\|\mathbf{r}\|)$

$V_{r}(r)=V_{r, \max } \begin{cases}\frac{r}{r_{\max }}, & 0<r<r_{\max } \\ \exp \left(-\frac{\left(r-r_{\max }\right)^{2}}{R_{r}^{2}}\right), & r \geq r_{\max }\end{cases}$

where $V_{r, \max }$ is the maximum radial velocity, $r_{\max }$ is the distance from the stagnation point and $R_{r}$ is a scaling length. The function $V_{r}(r)$ models the diffusive nature of the event, as well as the axisymmetric distribution of the radial velocity around the storm center.

Consequently, the time envelope $a(t)$ is determined by normalizing $\left\|\mathbf{V}_{r}+\mathbf{V}_{t}\right\|$ to one on $[0, T]$, with $\mathbf{V}_{t}=\left(V_{t}, 0\right)$ and $T$ being the duration of the phenomenon. The procedure to find the time envelope function ignores wind directionality. It is also the procedure proposed by Holmes and Oliver [33], and later used by Chen and Letchford [9] to analyze cantilever vertical structures and by Nguyen et al. [44] to examine slowly varying loads for wind turbine response in thunderstorm winds. In this application, we choose $V_{t}=12 \mathrm{~m} / \mathrm{s}, \quad r_{\max }=1000 \mathrm{~m}, V_{r, \text { max }}=47 \mathrm{~m} / \mathrm{s}, \quad R_{r}=700 \mathrm{~m}$, $d_{0}=-8000 \mathrm{~m}$ and $\ell=150 \mathrm{~m}$, as depicted in Fig. 6 .

A downburst presents a mean wind vertical profile different from the classic one [23], as it starts from zero, reaches a maximum at low altitude before slowly decreasing. It can be modeled by Wood's velocity profile $[9,70]$

$V(z)=1.55\left(\frac{z}{z_{0}}\right)^{\frac{1}{6}}\left(1-\operatorname{erf}\left(0.7 \frac{z}{z_{0}}\right)\right) V_{\max }$

with $z$ being the vertical coordinate. Therefore, the evolutionary mean wind speed reads $\bar{U}(t, z)=a(t) V(z)$. In the following, the parameter $z_{0}$ is chosen equal to $234.4 \mathrm{~m}$, while $V_{\max }$ depends on $V_{r, \max }$ and $V_{t}$.

The fluctuating part $\mathbf{u}(t)$ of the wind velocity is now described. Since data are quite rare, it is of common practice to use any available stationary PSD of wind speeds for the embedded stationary processes [9], as the von Karman PSD 

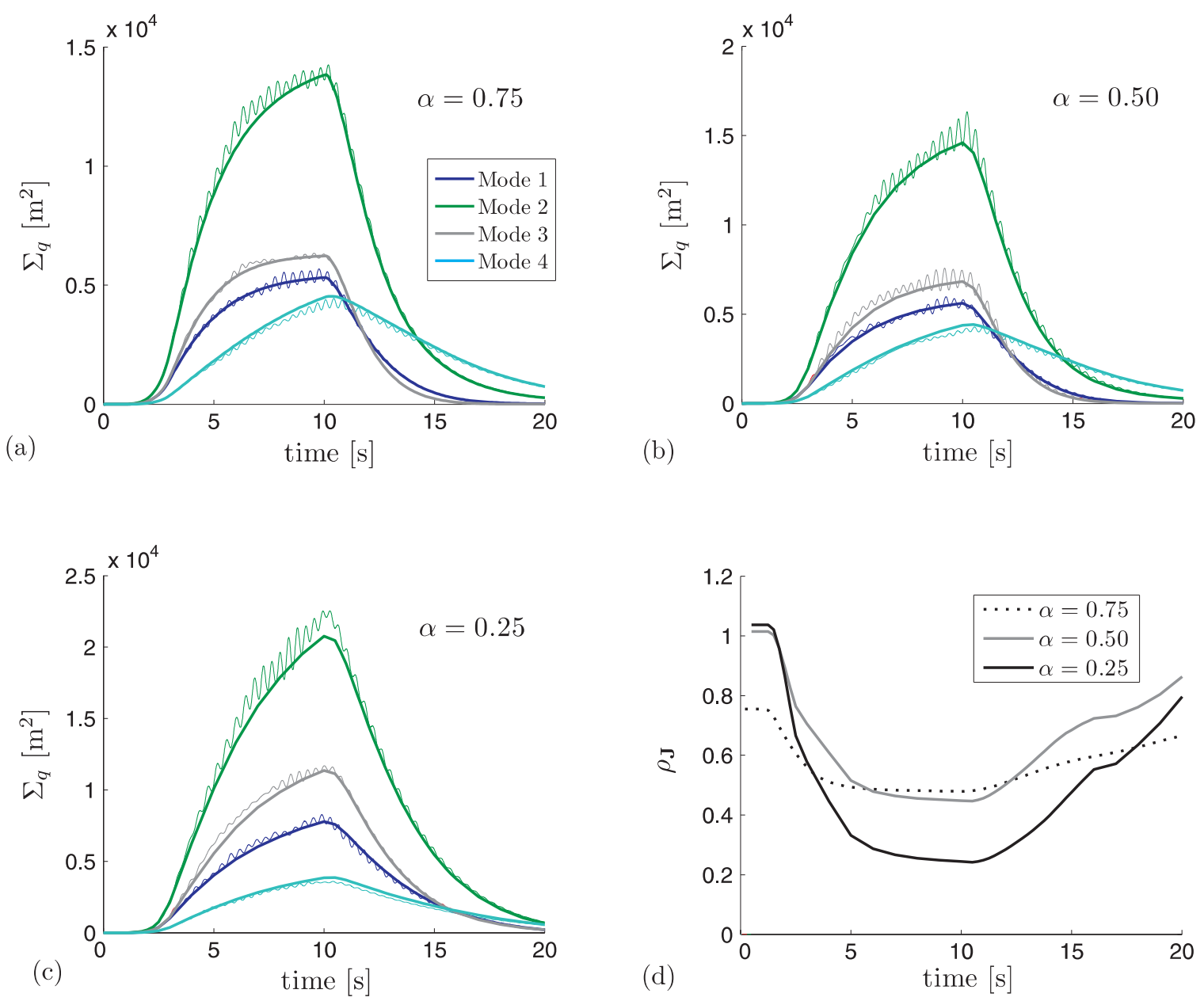

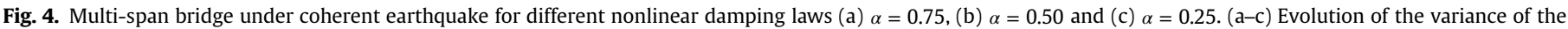

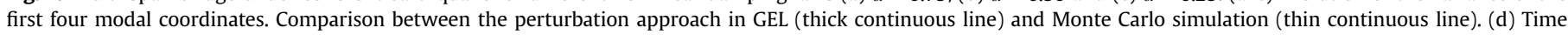
evolutions of the convergence criterion.

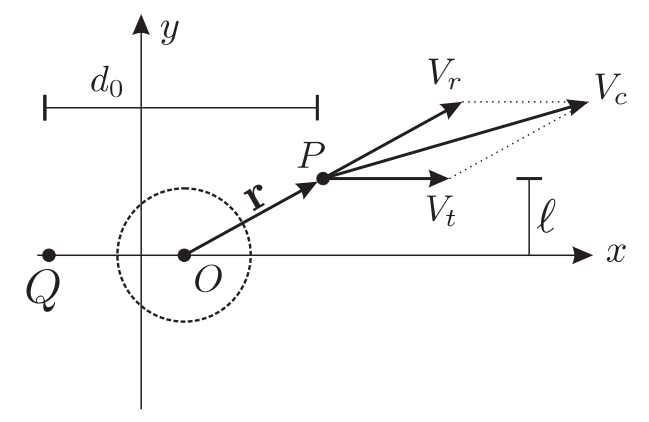

Fig. 5. Downburst model. Combination of radial and translation velocities.

$S_{u}(f, z)=\frac{4 L_{u}(z)}{V(z)} \frac{\sigma_{u}^{2}}{\left(1+70.78\left(\frac{f L_{u}(z)}{V(z)}\right)^{2}\right)^{\frac{5}{6}}}$

with $\sigma_{u}=I_{u} V(z)$ being the standard deviation of the wind turbulence, $I_{u}(z)$ the turbulence intensity with $I_{u}(z)=I_{u, 10}\left(\frac{10}{z}\right)^{\frac{1}{6}}$ $\left(I_{u, 10}=10 \%\right), \quad L_{u}(z)$ the turbulence length determined by
$L_{u}(z)=300\left(\frac{\max (z, 2)}{200}\right)^{0.52}$ according to [23] for a class 2 event. The coherence within the random wind field is usually modeled with an exponential decay as

$\Gamma\left(f, z_{i}, z_{j}\right)=\exp \left(-\frac{C_{r} f\left|z_{i}-z_{j}\right|}{\frac{1}{2}\left(V\left(z_{i}\right)+V\left(z_{j}\right)\right)}\right)$.

Thence, the cross-PSD of the wind velocity is build up as

$\left(\mathbf{S}_{\mathbf{u}}(f)\right)_{i j}=\sqrt{S_{u}\left(f, z_{i}\right) S_{u}\left(f, z_{j}\right)} \Gamma\left(f, z_{i}, z_{j}\right)$,

with $C_{r}=7$. The randomly fluctuating component of the wind now reads $\hat{\mathbf{u}}(t, z)=a(t) \mathbf{u}(t, z)$ with the distinction between $\mathbf{u}(t, z)$ the stationary process characterized by $\mathbf{S}_{\mathbf{u}}$ and the evolutionary process $\hat{\mathbf{u}}(t, z)$. The time envelope $a(t)$ is the same as the one defined for the mean wind velocity.

The evolutionary coherent wind load model is applied to a realistic structure inspired by [71,9]. The considered structure is a TV tower counting nine floors, depicted in Fig. 7a. The characteristics of inertia $E I_{i}$, wind surface $A_{i}$, mass $m_{i}$ and height $h_{i}$ are gathered in Table 1 . The drag coefficient is set equal to 0.7. A finite element model is built up with those characteristics. Every interstorey is divided into four elements to improve the numerical estimation of the coherence. The damping ratio of the undamped 


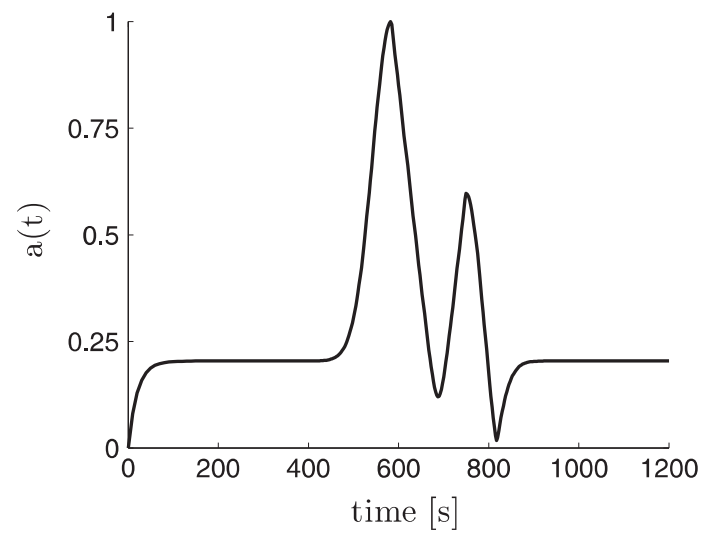

Fig. 6. Downburst model. Time envelope profile.

structure is set equal to $0.5 \%$ for the first and the third modes $(0.23$ and $0.92 \mathrm{~Hz}$, respectively).

On top floor of this structure, a Tuned Liquid Column Damper (TLCD) is set up in order to mitigate the vibrations of the tower. A TLCD is a nonlinear damping device, which controls the vibrations of a structure with the motion of liquid mass in a tube-like container [71], as depicted in Fig. 7b. The damping forces result from the head loss due to the presence of orifices in the container. The governing equation of the liquid elevation $v$ due to the horizontal displacement $x$ of the device [71] is the source of damping, modeled by

$\rho_{w} A_{w} L_{w} \ddot{v}+\frac{1}{2} \rho_{w} A_{w} \delta|\dot{v}| \dot{v}+2 \rho_{w} A_{w} g v=-\rho_{w} A_{w} B_{w} \ddot{x}$,

where $\rho_{w}, A_{w}, B_{w}$ and $L_{w}$ are the density, the cross-sectional area, the width and the length of the liquid column, respectively, while $\delta, g$ are the head loss coefficient and the acceleration of gravity, respectively. The statistically linearized equation, equivalent to (96), reads

$\rho_{w} A_{w} L_{w} \ddot{v}+\sqrt{\frac{2}{\pi}} \rho_{w} A_{w} \delta \sigma_{\dot{v}} \dot{v}+2 \rho_{w} A_{w} g v=-\rho_{w} A_{w} B_{w} \ddot{x}$.

The container is directly connected to the mass located at the top of the tower. The term $\rho_{w} A_{w} L_{w}$ is added to the lumped mass at the tower top and the coupling between this mass and the TLCD is modeled by the term $\rho_{w} A_{w} B_{w}$ in the mass matrix (see [71]). It may be worth observing that $\ddot{x}$ now becomes the acceleration of the rooftop node of the tower and that $\ddot{v}$ is modeled as an auxiliary
DOF. The parameters $\rho_{w}, A_{w}, L_{w}, B_{w}$ and $\delta$ are chosen equal to $1000 \mathrm{~kg} / \mathrm{m}^{3}, 1.18 \mathrm{~m}^{2}, 12.73 \mathrm{~m}, 0.9 L_{w}$, and 200 , respectively. Due to the installation of the TLCD, the natural frequencies of the first five structural modes become $0.17 \mathrm{~Hz}, 0.24 \mathrm{~Hz}, 0.36 \mathrm{~Hz}, 0.92 \mathrm{~Hz}$, and $1.38 \mathrm{~Hz}$, respectively.

In the following, the computational procedure developed in Section 3 is illustrated. The storm is simulated over a $1200 \mathrm{~s}$ time window, the time step is equal to $10 \mathrm{~s}$ for the first $400 \mathrm{~s}$ and after $1000 \mathrm{~s}$, while it is reduced to $5 \mathrm{~s}$ between these two intervals $(M=40+120+20=180)$.

The convergence is investigated on the structure with the TLCD in Fig. 8: the results obtained with $N=0$ are in good agreement with $N=2$, in spite of a small difference around the first peak, while the results obtained with $N=1$ and $N=2$ are superimposed, confirming the convergence of the expansion. The asymptotic expansion-based method and the stochastic linearization are compared with Monte Carlo simulations (600 samples) in Figs. 9 and 10. The transient equivalent linearization computed with our approach is in really good agreement with the simulations, validating the multiple scales assumptions, the use of equivalent linearization for TLCD and the proposed recurrence procedure.

In Fig. 10, we compare our multiple scales approach to the quasi-stationary hypothesis: the variance of the stationary response of the equivalent structure is readily modulated by the square of the time window. Fig. 10 shows that this assumption leads to satisfactory results, especially in terms of the maximum variance, but the time evolution is poorly captured.

Fig. 11 shows the time evolution of the convergence criterion $\rho_{\mathbf{J}}$, highlighting the strong coupling. It is noteworthy that the damper also reduces the duration of the maximum response. This is explained by the increase of the equivalent damping ratios as shown in Fig. 12. The damping ratio $\xi_{2}$ at time $600 \mathrm{~s}$ is at least multiplied by a factor 10 compared to the initial value of the uncontrolled structure. The damping ratio $\xi_{1}$ is more related to a local vibration mode of the top of the tower.

From a computational point of view, the resolution with the proposed approach has required about $80 \mathrm{~s}$ of cpu time (Intel Core i5) with $m=5$ and $N=1$. Table 2 compares different total cpu times required for different computations depending on the number of selected modes $m$ and the truncation order $N$. Those times are given, not as absolute references, but just to show that the developed method is absolutely not time consuming compared with Monte Carlo simulations (about one hour of computation for a resolution in a modal basis with 1000 samples). From Table 2, it seems that increasing the truncation order does not increase proportionally the

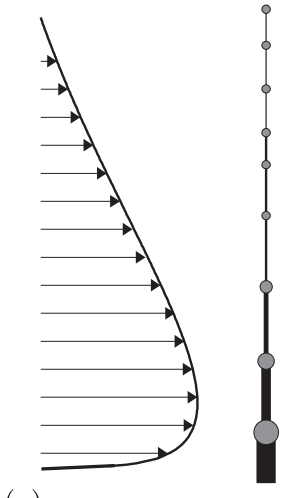

(a)

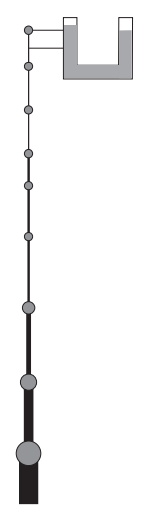

(b)

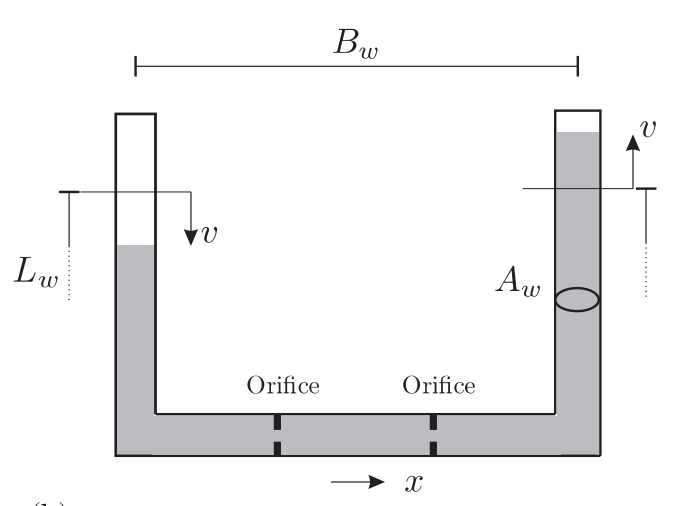

Fig. 7. (a) Tower subject to downburst with a TLCD set on the top. (b) Sketch of a TLCD. 
Table 1

Characteristics of the TV tower.

\begin{tabular}{|c|c|c|c|c|c|c|c|c|c|}
\hline Storey $i$ & $1 \mathrm{st}$ & 2nd & $3 r d$ & 4 th & 5 th & 6th & 7th & 8th & 9th \\
\hline$h_{i}(\mathrm{~m})$ & 20.00 & 28.00 & 28.00 & 27.25 & 19.25 & 12.50 & 17.75 & 17.75 & 14.25 \\
\hline$E I_{i}\left(10^{8} \mathrm{k} \mathrm{Nm}\right)$ & 145.35 & 47.23 & 25.47 & 7.41 & 4.13 & 1.06 & 0.27 & 0.02 & 0.01 \\
\hline$m_{i}$ (tons) & 6134 & 3853 & 2578 & 3032 & 692 & 85 & 72 & 51 & 23 \\
\hline$A_{i}(\mathrm{~m})$ & 489 & 460 & 320 & 474 & 125 & 75 & 49 & 24 & 10 \\
\hline
\end{tabular}

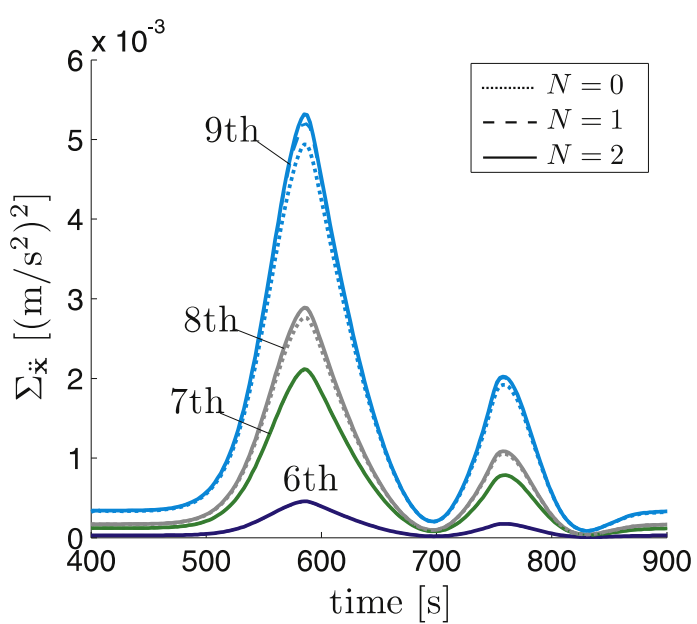

Fig. 8. Tower subject to downburst. Time evolution of the four upper floor accelerations. Influence of the truncation order. The results obtained for $N=1$ and $N=2$ are superimposed, despite a small difference around the first peak for the top floor.

computation time. In any case, the most consuming operation remains the computation of the integrals $I_{k}^{\alpha}$.

\section{Conclusions}

In civil engineering, evolutionary processes are commonly used to model transient phenomena. However, their time-frequency dual representation is very seldom employed, a reason for developing tools to improve evolutionary spectral analysis for both linear and nonlinear structures.

First, we developed a strategy in linear dynamics to approximate the IR matrix of a structure in the presence of modal coupling. Based on the Fourier duality and on an asymptotic expansion of the modal transfer matrix, we derived a closed-form expression of the asymptotic expansion of the IR matrix. Then, invoking the semi-group property, we found a recurrence relation to compute the StSpEvTr matrix of the modal coordinates, time interval by interval. This relation enables to deal with different time windows and to take advantage of the convergence rate of the asymptotic expansion of the IR matrix, improved on short time intervals. Therefore, this convergence rate may be enhanced by increasing the number of the time intervals, instead of increasing the number of correction terms.

In nonlinear dynamics, we used the GEL to extend the previous results. This method transforms the initial nonlinear time-invariant system into a linear time-variant one. Within the system, we emphasized the presence of a fast structural timescale and a slow statistical one, enabling the theoretical use of a multiple scales approach. Consequently, the linearized system may be considered to respond as a piecewise time-invariant system, as the equivalent structural matrices mainly depends on the slow timescale. The gap

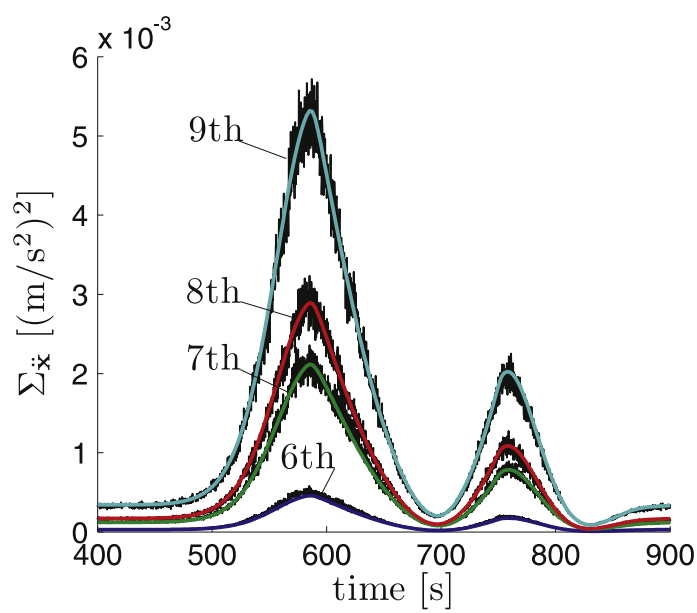

Fig. 9. Tower subject to downburst. Time evolution of the four upper floor accelerations. Comparison of the asymptotic expansion-based method $(N=2)$ with Monte Carlo simulation.

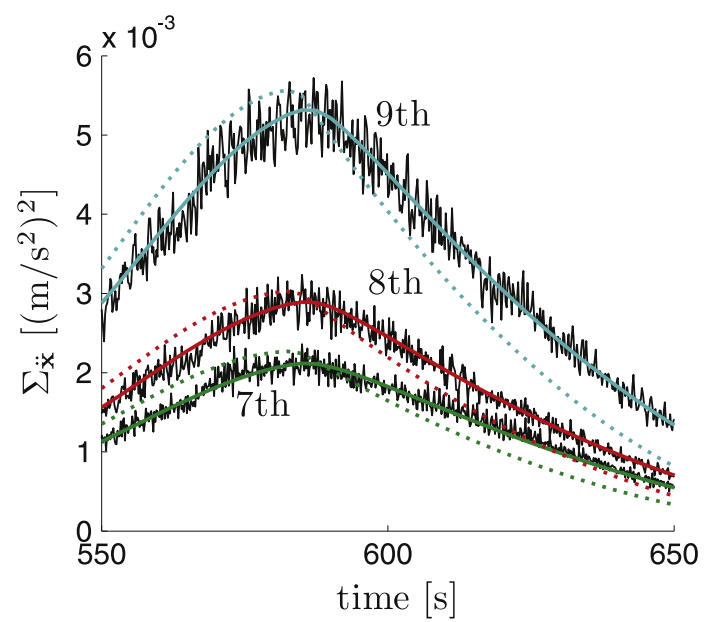

Fig. 10. Tower subject to downburst. Time evolution of the three upper floor accelerations. Comparison of the asymptotic expansion-based method $(N=2)$ with Monte Carlo simulation and with the quasi-stationary approach (dotted lines).

between the GEL and the EvSp analysis can be overcome, since the asymptotic expansion-based method and the recurrence relation can be applied, by simply adapting, piecewisely, the equivalent structural matrices.

Our developments were then applied to examples in wind and earthquake engineering, i.e. to nonlinear structures subject to evolutionary and partially coherent random loads. We can highlight the good agreement between our method and Monte Carlo simulation, as well as its computational efficiency for second-order statistical analysis. 


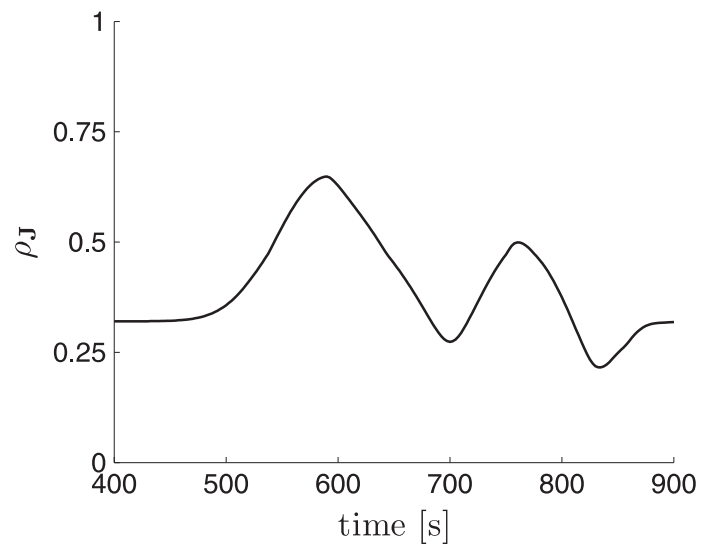

Fig. 11. Tower subject to downburst. Time evolution of the convergence criterion $\rho_{\mathbf{J}} \cdot$

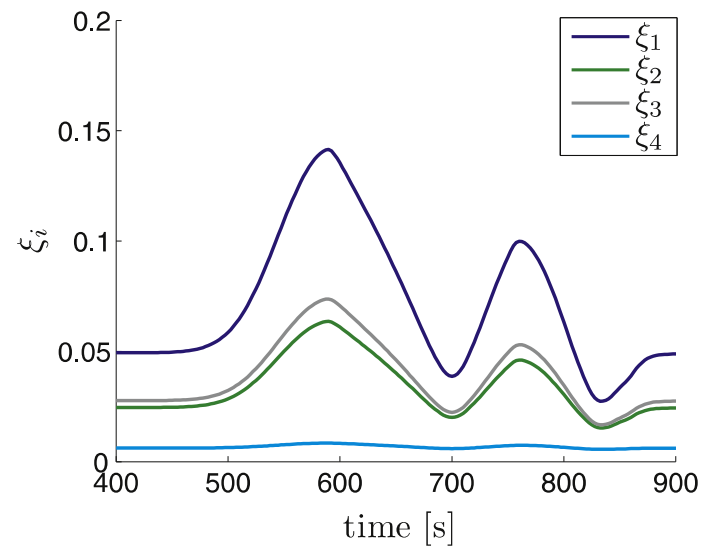

Fig. 12. Tower subject to downburst. Time evolution of the modal damping ratios.

Table 2

Tower subject to downburst. Computating time for different truncation orders $N$ and numbers of modes $m$ (to be compared with Monte Carlo Simulations: approx. $1 \mathrm{~h}$ for 1000 simulations).

\begin{tabular}{llll}
\hline Number of modes & $N=0$ & $N=1$ & $N=2$ \\
\hline$m=5$ & $78 \mathrm{~s}$ & $80 \mathrm{~s}$ & $94 \mathrm{~s}$ \\
$m=7$ & $115 \mathrm{~s}$ & $127 \mathrm{~s}$ & $167 \mathrm{~s}$ \\
\hline
\end{tabular}

\section{Acknowledgments}

The first author is grateful to the National Fund for Scientific Research (F.R.S.-FNRS) of Belgium for its support. He also would like to personally acknowledge Angelo Mariani for his kind attention and his precious advices. They will never be forgotten.

\section{References}

[1] S. Ates, Seismic behaviour of isolated multi-span continuous bridge to nonstationary random seismic excitation, Nonlinear Dyn. 67 (1) (2012) 263-282.

[2] M. Bartlett, An Introduction to Stochastic Processes, 1st ed., Cambridge University Press, Cambridge, 1956.

[3] G.Q. Cai, Y. Suzuki, Response of systems under non-Gaussian random excitations, Nonlinear Dyn. 45 (1-2) (2006) 95-108.

[4] T. Canor, New perspectives on probabilistic methods for nonlinear transient dynamics in civil engineering (Ph.D. thesis), Université de Liège, Liège, 〈http:// hdl.handle.net/2268/166823〉, 2014.

[5] T. Canor, N. Blaise, V. Denoël, Efficient uncoupled stochastic analysis with nonproportional damping, J. Sound Vib. 331 (2012) 5283-5291.

[6] T. Canor, N. Blaise, V. Denoël, An asymptotic expansion-based method for a spectral approach in equivalent statistical linearization, Probab. Eng. Mech. (2014) 〈http://hdl.handle.net/10.1016/j.probengmech.2014.08.003〉.

[7] T. Caughey, Response of a nonlinear string to random loading, J. Appl. Mech. (ASME) 26 (1959) 341-344.

[8] T. Caughey, Response of van der Pol's oscillator to random loading. J. Appl. Mech. (ASME) 26 (1959) 345-348.

[9] L. Chen, C. Letchford, A deterministic-stochastic hybrid model of downbursts and its impact on a cantilevered structure, Eng. Struct. 26 (5) (2004) 619-629.

[10] L. Chen, C. Letchford, Numerical simulation of extreme winds from thunderstorm downbursts, J. Wind Eng. Ind. Aerodyn. 9 (2007) 977-990.

[11] X. Chen, Analysis of along wind tall building response to transient nonstationary winds, J. Struct. Eng. 134 (5) (2008) 782-791.

[12] R.W. Clough, J. Penzien, Dynamics of Structures, 2nd ed., McGraw-Hill, New York, 1993.

[13] J.P. Conte, B.F. Peng, An explicit closed-form solution for linear systems subjected to nonstationary random excitation, Probab. Eng. Mech. 11 (1) (1996) 37-50.

[14] A. Cunha, The role of the stochastic equivalent linearization method in the analysis of the non-linear seismic response of building structures, Earthq. Eng. Struct. Dyn. 23 (8) (1994) 837-857.

[15] V. Denoël, Multiple timescale spectral analysis, Probab. Eng. Mech. 39 (2015) 69-86.

[16] V. Denoël, H. Degée, Asymptotic expansion of slightly coupled modal dynamic transfer functions, J. Sound Vib. 328 (2009) 1-8.

[17] V. Denoël, E. Detournay, Multiple scales solution for a beam with a small bending stiffness, J. Eng. Mech. 136 (1) (2010) 69-77.

[18] A. Der Kiureghian, A coherency model for spatially varying ground motion, Earthq. Eng. Struct. Dyn. 25 (1) (1996) 99-111.

[19] M. Di Paola, Digital simulation of wind field velocity, J. Wind Eng. Ind. Aerodyn. 74-76 (0) (1998) 91-109.

[20] C Dyrbye, S Hansen, Wind Loads on Structures, 1st ed., John Wiley and Sons New York, 1997.

[21] S.A. Emam, A.H. Nayfeh, On the nonlinear dynamics of a buckled beam subjected to a primary-resonance excitation, Nonlinear Dyn. 35 (1) (2004) 1-17.

[22] E. Esmaeilzadeh Seylabi, H. Jahankhah, M. Ali Ghannad, Equivalent linearization of non-linear soil-structure systems, Earthq. Eng. Struct. Dyn. 41 (13) (2012) 1775-1792.

[23] Eurocode1-1991-1-4 (1991) Eurocode 1: Actions on Structures Part 1-4: General Actions: Wind Actions, CEN.

[24] F. Gani, F. Légeron, Relationship between specified ductility and strength demand reduction for single degree-of-freedom systems under extreme wind events, J. Wind Eng. Ind. Aerodyn. 109 (0) (2012) 31-45.

[25] M. Grigoriu, Stochastic Calculus. Applications in Science and Engineering, 1st ed., Birkhauser, Boston, 2002.

[26] T.K. Guha, R.N. Sharma, P.J. Richards, Internal pressure in a building with multiple dominant openings in a single wall: comparison with the single opening situation, J. Wind Eng. Ind. Aerodyn. 107-108 (0) (2012) 244-255.

[27] I.D. Gupta, M.D. Trifunac, A note on the nonstationarity of seismic response of structures, Eng. Struct. 22 (11) (2000) 1567-1577.

[28] J.K. Hammond, On the response of single and multidegree of freedom systems to non-stationary random excitations, J. Sound Vib. 7 (1968) 393-416.

[29] J.K. Hammond, Evolutionary spectra in random vibrations, J. R. Stat. Soc. Ser. B (Methodol.) 35 (2) (1973) 167-188.

[30] R.S. Harichandran, E.H. Vanmarcke, Stochastic variation of earthquake ground motion in space and time, J. Eng. Mech. 112 (2) (1986) 154-174.

[31] J. Hespanha, Linear Systems Theory, Princeton University Press, Oxford, 2009.

[32] E. Hinch, Perturbation Methods, vol. 1, Cambridge University Press, Cambridge, 1991.

[33] J.D. Holmes, S.E. Oliver, An empirical model of a downburst, Eng. Struct. 22 (9) (2000) 1167-1172.

[34] R Horn, J CR, Matrix Analysis, 2nd ed., Cambridge University Press, Cambridge, 2013.

[35] M. Kahan, Approches stochastiques pour le calcul des ponts aux séismes (Ph.D thesis), Ecole Nationale des Ponts et Chaussées, Paris, 1996.

[36] I. Kougioumtzoglou, P. Spanos, Nonlinear MDOF system stochastic response determination via a dimension reduction approach, Comput. Struct. 126 (0) (2013) 135-148.

[37] E. Kreyszig, Advanced Engineering Mathematics, 9th ed., John Wiley and Sons, Inc., New York, 2006.

[38] J. Lin, W. Zhang, F.W. Williams, Pseudo-excitation algorithm for nonstationary random seismic responses, Eng. Struct. 16 (4) (1994) 270-276.

[39] Y. Lin, G. Cai, Advanced theory and applications, Probabilistic Structural Dynamics, 2nd ed., McGraw-Hill, New York, 2004.

[40] A. Luongo, A. Paolone, On the reconstitution problem in the multiple timescale method, Nonlinear Dyn. 19 (2) (1999) 135-158.

[41] M. Morzfeld, N. Ajavakom, F. Ma, Diagonal dominance of damping and the decoupling approximation in linear vibratory systems, J. Sound Vib. 320 (2009) 406-420.

[42] G.P. Nason, Sachs. Rv, G. Kroisandt, Wavelet processes and adaptive estimation of the evolutionary wavelet spectrum, J. R. Stat. Soc. Ser. B (Stat. Methodol.) 62 (2) (2000) 271-292.

[43] A.H. Nayfeh, Pure and applied mathematics, Perturbation Methods, J. Wiley \& Sons, New York, 1973. 
[44] H. Nguyen, L. Manuel, J. Jonkman, P. Veers, Simulation of thunderstorm downbursts and associated wind turbine loads, J. Sol. Energy Eng.-Trans. ASME 135 (2) (2013) 014-021.

[45] F. Perotti, Structural response to non-stationary multiple-support random excitation, Earthq. Eng. Struct. Dyn. 19 (1990) 513-527.

[46] H. Pradlwarter, G. Schuëller, C. Schenk, A computational procedure to estimate the stochastic dynamic response of large non-linear fe-models, Comput. Methods Appl. Mech. Eng. 192 (2003) 777-801.

[47] A. Preumont, Random Vibration and Spectral Analysis, Kluwer Academic Publishers, Dordrecht, The Netherlands, 1994.

[48] M.B. Priestley, Evolutionary spectra and non-stationary processes, J. R. Stat. Soc. Ser. B (Methodol.) 27 (2) (1965) 204-237.

[49] M.B. Priestley, Power spectral analysis of non-stationary random processes, J. Sound Vib. 6 (1967) 86-97.

[50] C. Proppe, H.J. Pradlwarter, G.I. Schuëller, Equivalent linearization and MonteCarlo simulation in stochastic dynamics, Probab. Eng. Mech. 18 (1) (2003) $1-15$.

[51] J. Rayleigh, The Theory of Sound, vol. 1, Dover Publication, New York, 1945.

[52] J Roberts, P Spanos, Random Vibration and Statistical Linearization, 1st ed., Dover Pub. Inc., Mineola, NY, 1999.

[53] H.W. Rong, G. Meng, W. Xu, T. Fang, Response statistics of three-degree-offreedom nonlinear system to narrow-band random excitation, Nonlinear Dyn. 32 (1) (2003) 93-107.

[54] C.A. Schenk, H.J. Pradlwarter, G.I. Schuëller, On the dynamic stochastic response of fe models, Probab. Eng. Mech. 19 (1-2) (2004) 161-170.

[55] C.A. Schenk, H.J. Pradlwarter, G.I. Schuëller, Non-stationary response of large, non-linear finite element systems under stochastic loading, Comput. Struct. 83 (14) (2005) 1086-1102.

[56] A. Sengupta, F. Haan, P. Sarkar, V. Balaramudu, Transient loads on buildings in microburst and tornado winds, J. Wind Eng. Ind. Aerodyn. 96 (10-11) (2008) 2173-2187.

[57] K. Shaska, R.A. Ibrahim, R.F. Gibson, Influence of excitation amplitude on the characteristics of nonlinear butyl rubber isolators, Nonlinear Dyn. 47 (1-3) (2007) 83-104.

[58] M. Shinozuka, Y. Sato, Simulation of nonstationary random processes, J. Eng. Mech. Div. 93 (1) (1967) 11-40.

[59] M. Shinozuka, G. Deodatis, R. Zhang, A. Papageorgiou, Modeling, synthetics and engineering applications of strong earthquake wave motion, Soil Dyn.
Earthq. Eng. 18 (3) (1999) 209-228.

[60] A. Smyth, S. Masri, Nonstationary response of nonlinear systems using equivalent linearization with a compact analytical form of the excitation process, Probab. Eng. Mech. 17 (1) (2002) 97-108.

[61] G. Solari, G. Piccardo, Probabilistic 3-D turbulence modeling for gust buffeting of structures, Probab. Eng. Mech. 16 (2001) 73-86.

[62] P.D. Spanos, I.A. Kougioumtzoglou, Harmonic wavelets based statistical linearization for response evolutionary power spectrum determination, Probab. Eng. Mech. 27 (1) (2012) 57-68.

[63] A.A. Taflanidis, G. Jia, A simulation-based framework for risk assessment and probabilistic sensitivity analysis of base-isolated structures, Earthq. Eng. Struct. Dyn. 40 (14) (2011) 1629-1651.

[64] C. To, The stochastic central difference method in structural dynamics, Comput. Struct. 23 (1986) 813-818.

[65] C. To, A stochastic version of the newmark family of algorithms for discretized dynamic systems, Comput. Struct. 44 (3) (1992) 667-673.

[66] M. Tootkaboni, L. Graham-Brady, Stochastic direct integration schemes for dynamic systems subjected to random excitations, Probab. Eng. Mech. 25 (2) (2010) 163-171.

[67] E. Tubaldi, I.A. Kougioumtzoglou, Nonstationary stochastic response of structural systems equipped with nonlinear viscous dampers under seismic excitation, Earthq. Eng. Struct. Dyn. 44 (1) (2015) 121-138.

[68] D.D. Vicroy, Assessment of microburst models for downdraft estimation, J. Airc. 29 (1992) 1043-1048.

[69] A.Y.J. Won, J.A. Pires, M.A. Haroun, Stochastic seismic performance evaluation of tuned liquid column dampers, Earthq. Eng. Struct. Dyn. 25 (11) (1996) 1259-1274.

[70] G. Wood, K. Kwok, An empirically derived estimate for the mean velocity profile of a thunderstorm downburst, in: Seventh AWES Workshop, Auckland, New Zealand, 1998.

[71] Y. Xu, B. Samali, K. Kwok, Control of along-wind response of structures by mass and liquid dampers, J. Eng. Mech. 118 (1992) 20-39.

[72] A. Zerva, V. Zerva, Spatial variation of seismic ground motions: an overview, Appl. Mech. Rev. (ASME) 55 (3) (2002) 271-297.

[73] Y. Zhang, H. Hu, P. Sarkar, Modeling of microburst outflows using impinging jet and cooling source approaches and their comparison, Eng. Struct. 56 (2013) 779-793. 